\title{
Hic-5 mediates the initiation of endothelial sprouting by regulating a key surface metalloproteinase
}

\author{
Jui M. Dave ${ }^{1, *}$, Colette A. Abbey ${ }^{1, *}$, Camille L. Duran ${ }^{1}$, Heewon Seo², Gregory A. Johnson² and Kayla J. Bayless ${ }^{1, \ddagger}$
}

\begin{abstract}
During angiogenesis, endothelial cells must coordinate matrix proteolysis with migration. Here, we tested whether the focal adhesion scaffold protein Hic-5 (also known as TGFB1I1) regulated endothelial sprouting in three dimensions. Hic-5 silencing reduced endothelial sprouting and lumen formation, and sprouting defects were rescued by the return of Hic- 5 expression. Pro-angiogenic factors enhanced colocalization and complex formation between membrane type-1 matrix metalloproteinase (MT1-MMP, also known as MMP14) and Hic-5, but not between paxillin and MT1-MMP. The LIM2 and LIM3 domains of Hic-5 were necessary and sufficient for Hic-5 to form a complex with MT1-MMP. The degree of interaction between MT1-MMP and Hic-5 and the localization of the complex within detergent-resistant membrane fractions were enhanced during endothelial sprouting, and Hic-5 depletion lowered the surface levels of MT1-MMP. In addition, we observed that loss of Hic-5 partially reduced complex formation between MT1-MMP and focal adhesion kinase (FAK, also known as PTK2), suggesting that Hic-5 bridges MT1-MMP and FAK. Finally, Hic-5 LIM2-LIM3 deletion mutants reduced sprout initiation. Hic-5, MT1-MMP and FAK colocalized in angiogenic vessels during porcine pregnancy, supporting that this complex assembles during angiogenesis in vivo. Collectively, Hic-5 appears to enhance complex formation between MT1-MMP and FAK in activated endothelial cells, which likely coordinates matrix proteolysis and cell motility.
\end{abstract}

KEY WORDS: Collagen, Endothelial cell, Angiogenesis, Threedimensional, Cell migration, Matrix degradation, MT1-MMP, FAK, S1P, LIM

\section{INTRODUCTION}

Angiogenesis is defined as new blood vessel growth from preexisting structures (Folkman and D'Amore, 1996; Carmeliet, 2003). During angiogenesis, quiescent endothelial cells are activated in response to external pro-angiogenic cues to proliferate, migrate, invade and form lumens within the surrounding three-dimensional (3D) matrix (Adams and Alitalo, 2007; Iruela-Arispe and Davis, 2009). Loss of angiogenic regulation is a common denominator in several human disorders, including, but not limited to, cancer (Folkman, 1995), rheumatoid arthritis (Azizi et al., 2014) and macular degeneration (Ambati and Fowler, 2012). Thus, understanding the molecular signaling pathways that regulate endothelial cell activation and sprouting is

\footnotetext{
${ }^{1}$ Department of Molecular and Cellular Medicine, Texas A\&M Health Science Center, College Station, TX 77843, USA. ${ }^{2}$ Veterinary Medicine and Biomedical Sciences, Texas A\&M University, College Station, TX 77843, USA.

*These authors contributed equally to this work

${ }^{\ddagger}$ Author for correspondence (kbayless@medicine.tamhsc.edu)
}

Received 23 February 2015; Accepted 5 January 2016 crucial to gain further insights into the events that regulate angiogenesis.

In this study, we investigated whether the $55-\mathrm{kDa}$ focal adhesion scaffold protein, hydrogen-peroxide-inducible clone 5 (Hic-5, also known as TGFB1I1), regulated endothelial sprouting events in 3D matrices. Hic-5 is expressed in endothelial cells (Kim-Kaneyama, 2012) and, although Hic-5-knockout mice appear to be normal and fertile, subsequent studies have revealed defective recovery following vascular injury of the femoral artery (Kim-Kaneyama et al., 2011). Hic-5 was originally discovered by differential screening of a cDNA library of genes induced by transforming growth factor $\beta$ (TGF $\beta$ ) (Shibanuma et al., 1994). Hic-5 is highly similar to paxillin and contains four N-terminal Leu- and Asp-rich (LD) domains and four C-terminal LIM domains. The N-terminal LD domains of Hic-5 interact with focal adhesion kinase (FAK, also known as PTK2) (Fujita et al., 1998) vinculin (Deakin et al., 2012), paxillin (Deakin et al., 2012), c-Src kinase (Csk) (Thomas et al., 1999), protein tyrosine kinase $2 \beta$ (PYK2, also known as PTK2B) (Matsuya et al., 1998) and Arf GAP1 (GIT1) (Nishiya et al., 2002). The LIM domains at the C-terminus of Hic-5 mediate binding to PTP-PEST (also known as PTPN12) (Nishiya et al., 1999) and Hsp27 (also known as HSPB1) (Jia et al., 2001). Oxidative stress induces Hic-5 translocation into the nucleus, where it acts as a transcriptional coactivator (Shibanuma et al., 2002, 2003, 2004). Thus, Hic-5 is a mobile scaffold protein that interacts with several proteins at focal adhesion sites and shuttles from the cytoplasm to the nucleus.

Recent studies have established a link between Hic-5 and invadopodia, which were first identified as dot-shaped actin-rich membrane protrusions that form in cells seeded on two-dimensional substrates (Artym et al., 2006). Hic-5 localizes to pseudopodia in bovine pulmonary artery endothelial cells (Avraamides et al., 2007) and invadopodia of breast cancer cells (Pignatelli et al., 2012). Studies in breast cancer cells have revealed that Hic-5 silencing attenuates integrin-mediated adhesion, migration, invasion and tumor cell metastasis (Deakin et al., 2012), and causes defective matrix degradation (Pignatelli et al., 2012). In addition to Hic-5, invadopodia also contain membrane type-1 matrix metalloproteinase (MT1-MMP, also known as MMP14) (Egeblad and Werb, 2002). MT1-MMP is a member of the MMP family that contains a transmembrane domain, which anchors it to the cell surface and facilitates the proximal extracellular matrix (ECM) breakdown needed for invasion into 3D matrices (Hotary et al., 2000; Yana and Weiss, 2000; Uekita et al., 2001; Egeblad and Werb, 2002; Li et al., 2008). Although multiple classes of MMPs have been implicated in angiogenic events, MT1-MMP is essential for endothelial invasion through a 3D ECM. Unlike mice null for MMP-2 or MMP-9, neovessel formation and collagenolysis are completely inhibited in MT1-MMP-null tissue explants and microvascular cells (Chun et al., 2004), indicating that MT1-MMP is needed for endothelial cells to remodel and invade the ECM. In vivo, MT1-MMP localizes to 
sprouting tip cells (Yana et al., 2007). MT1-MMP is also required for lumen formation in vitro. Overexpression of wild-type MT1-MMP increases lumen formation, whereas MT1-MMP small interfering RNA (siRNA) blocks invasion and lumen formation (Stratman et al., 2009). Finally, MT1-MMP gene disruption causes abnormalities in osteogenesis, FGF-induced angiogenesis and collagen turnover, leading to reduced neovessel formation, dwarfism, arthritis and premature death in knockout mice (Holmbeck et al., 1999; Zhou et al., 2000). Taken together, these data support a crucial role for MT1-MMP in endothelial cell invasion of the ECM. Although previous studies have suggested that Hic-5, as well as MT1-MMP, regulates tumor cell migration, invasion, adhesion and matrix degradation, Hic-5 has not been implicated in endothelial sprouting responses in a 3D environment, nor has a link been established between MT1-MMP and Hic-5. Here, we show for the first time that Hic-5 participates in MT1-MMP-dependent endothelial sprout initiation by enhancing complex formation between MT1-MMP and FAK in response to endothelial cell activation.

\section{RESULTS}

Hic-5 silencing decreased S1P- and growth-factor-mediated endothelial cell invasion and lumen formation in 3D matrices

To test the effect of Hic-5 depletion on endothelial sprouting, non-transduced endothelial cells (wild type, WT), or endothelial cells transduced with recombinant lentiviruses from individual short hairpin RNA (shRNA) sequences directed against $\beta 2$ microglobulin $(\operatorname{sh} \beta 2 \mathrm{M})$ or Hic-5 (shHic-5-1 and -2$)$ were utilized in an established model of endothelial cell invasion (Bayless et al., 2009). To rule out off-target effects, mRNA and protein levels were monitored for several molecules that are known to regulate angiogenesis, and no major changes in any of the molecules tested were observed (Fig. S1). Hic-5 silencing in endothelial cells did not result in cell toxicity or adversely affect cell morphology (data not shown). Images of invading cultures illustrated that knockdown of Hic-5 with two individual shRNA sequences interfered with endothelial cell invasion responses (Fig. 1A). Western blot analyses of extracts collected from invading cultures confirmed knockdown of Hic-5 and $\beta 2 \mathrm{M}$ by their respective shRNAs (Fig. 1B). Quantification of invasion density revealed a significantly decreased number of invading cells with Hic-5 silencing compared to $\operatorname{sh} \beta 2 \mathrm{M}$ and WT controls (Fig. 1C). In addition, the invasion distance from the cell monolayer was significantly reduced with Hic-5 silencing compared to both $\operatorname{sh} \beta 2 \mathrm{M}$ and WT controls (Fig. 1D). Side view images of invading cultures illustrated that knockdown of Hic-5 resulted in smaller lumens compared to WT or $\operatorname{sh} \beta 2 \mathrm{M}$ controls (Fig. 1E). Quantification of the lumen size revealed a significant decrease in lumen diameter with Hic-5 knockdown (Fig. 1F). In addition, the number of invading structures forming lumens was decreased with Hic-5 silencing compared to WT and $\operatorname{sh} \beta 2 \mathrm{M}$ controls (Fig. 1G). In rescue experiments, expression of Hic-5 resulted in invasion responses resembling WT endothelial cells (Fig. 1H). Protein expression of full-length Hic-5 was confirmed by western blotting (Fig. 1I), and GFP expression was confirmed visually (data not shown). Quantification of invasion responses revealed a significant improvement in invasion density (Fig. 1J), invasion distance (Fig. 1K) and lumen formation (Fig. 1L) upon Hic-5 rescue compared to GFP controls. These results demonstrated that Hic-5 participates in endothelial cell invasion and successful lumen formation in 3D collagen matrices.

\section{Pro-angiogenic factors enhanced complex formation} between Hic-5 and MT1-MMP in endothelial cells

Fig. 1 shows that Hic-5 silencing interfered with sprout initiation and lumen formation in endothelial cells stimulated with sphingosine 1-phosphate (S1P) and growth factors [40 ng/ml VEGF and basic FGF (bFGF)], which are both required to promote robust endothelial cell invasion (Fig. S2A). Endothelial lumen formation is a crucial aspect of endothelial morphogenesis and tubulogenesis (Davis et al., 2011), and MT1-MMP is required for both successful lumen formation and endothelial cell sprouting into collagen matrices (Chun et al., 2004; Stratman et al., 2009; Sacharidou et al., 2010). S1P has been shown to stimulate phosphorylation of MT1-MMP at Y573 (Nyalendo et al., 2007). Because a link between MT1-MMP and Hic-5 has not been established, we first investigated whether Hic-5 colocalized with phosphorylated MT1-MMP (pMT1-MMP Y573) in endothelial cells in response to activating stimuli. Endothelial cells seeded on coverslips were serum starved, treated with nothing (Control), $40 \mathrm{ng} / \mathrm{ml}$ of growth factors (GF), $1 \mu \mathrm{M} \mathrm{S1P}$ (S1P), or S1P and growth factors $(\mathrm{S} 1 \mathrm{P}+\mathrm{GF})$ for $1 \mathrm{~h}$ and stained with antibodies directed against Hic-5 or pMT1-MMP (Y573). Overlay images indicated enhanced colocalization between Hic-5 and pMT1-MMP in endothelial cells treated with $\mathrm{S} 1 \mathrm{P}+\mathrm{GF}$ (Fig. 2A), although S1P and growth factor treatments alone induced slight colocalization. These results suggest that $\mathrm{S} 1 \mathrm{P}+\mathrm{GF}$ stimulation promotes increased Hic-5 and MT1-MMP colocalization that coincides with the maximal sprouting response (Fig. S2A). Importantly, western blot analyses demonstrated that pMT1-MMP antibodies reacted with the correct molecular mass $(60 \mathrm{kDa})$ protein (Fig. S2B) and immunoprecipitated MT1-MMP (Fig. S2C).

To confirm whether $\mathrm{S} 1 \mathrm{P}+\mathrm{GF}$ stimulated complex formation between Hic-5 and MT1-MMP, immunoprecipitations were performed with or without endothelial cell activation using MT1MMP-specific or control (IgG) antisera. We observed increased Hic-5 and MT1-MMP association with S1P+GF stimulation (Fig. 2B). Because Hic-5 is closely related to paxillin, we also tested whether paxillin might associate with MT1-MMP following $\mathrm{S} 1 \mathrm{P}+\mathrm{GF}$ stimulation (Fig. 2C) or in the absence of Hic-5 (Fig. 2D). Using identical conditions, we were unable to detect an association between MT1-MMP and paxillin, indicating endothelial cell activation with $\mathrm{S} 1 \mathrm{P}+\mathrm{GF}$ selectively enhanced complex formation between MT1-MMP and Hic-5, but not paxillin.

\section{The LIM2 and LIM3 domains of Hic-5 mediate its interaction with MT1-MMP}

The above data suggest that a complex forms between Hic-5 and MT1-MMP in endothelial cells. We next determined which domains within Hic-5 mediate the formation of this complex (see Fig. 3A for constructs used). In initial experiments, MT1-MMP was co-transfected with full-length (FL) Hic-5, N-terminal Hic-5 (N), C-terminal Hic-5 (C), or GFP in HEK-293 cells (Fig. 3B). Immunoprecipitation experiments were performed by incubating lysates with MT1-MMP-specific or control (IgG) antisera. Western blot analyses revealed successful expression of MT1-MMP, GFP and Hic-5 constructs (Input). We observed that MT1-MMP formed complexes with full-length and C-terminal Hic-5 constructs, but not the N-terminal Hic-5 construct (Fig. 3B), suggesting that the LIM domains within the C-terminus of Hic-5 are involved in formation of the complex with MT1-MMP. To identify the specific C-terminal domain(s) involved in MT1-MMP complex formation, the LIM domains of Hic-5 were either truncated or deleted, and the resulting constructs were used in immunoprecipitation experiments. MT1- 

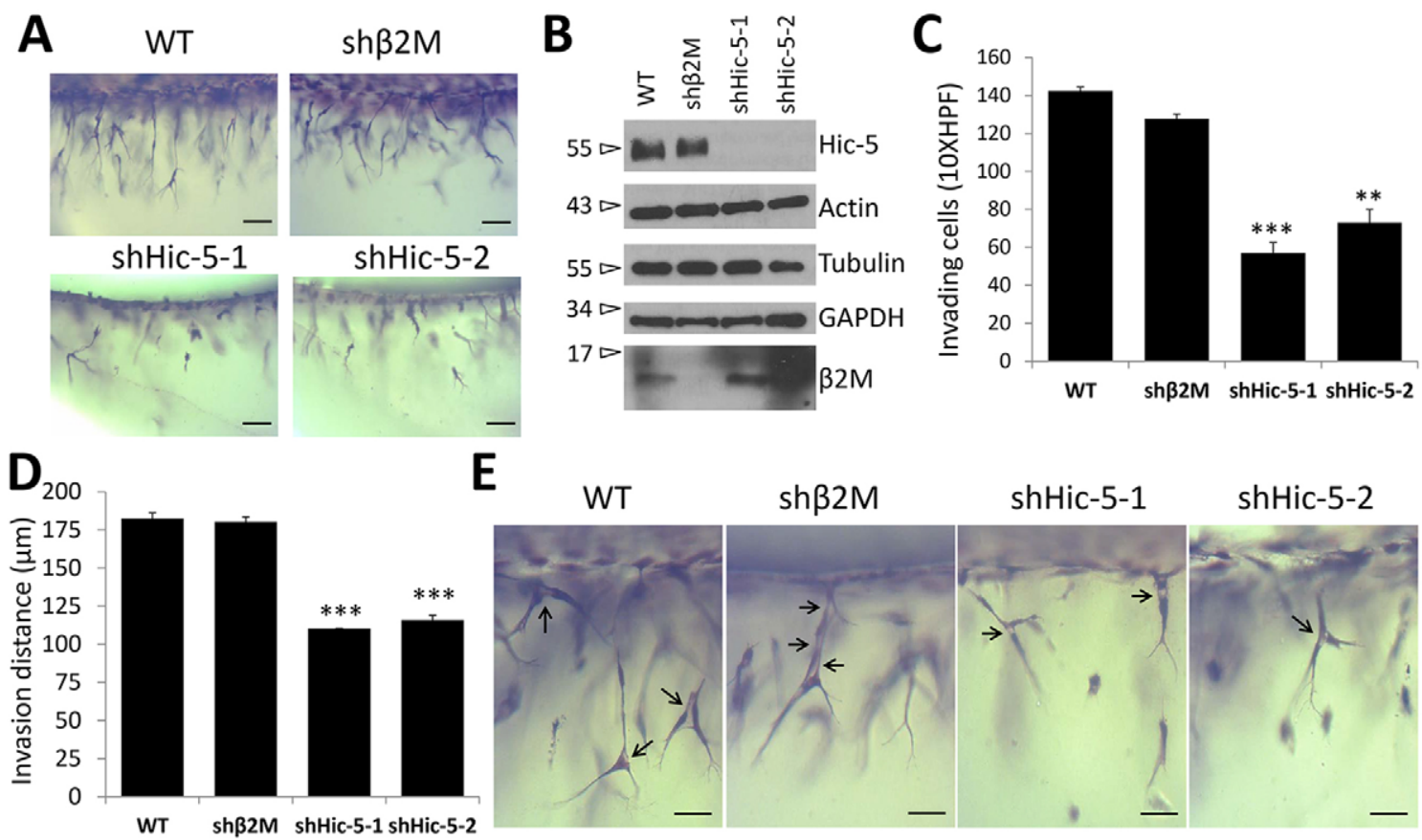

$\mathbf{E}$
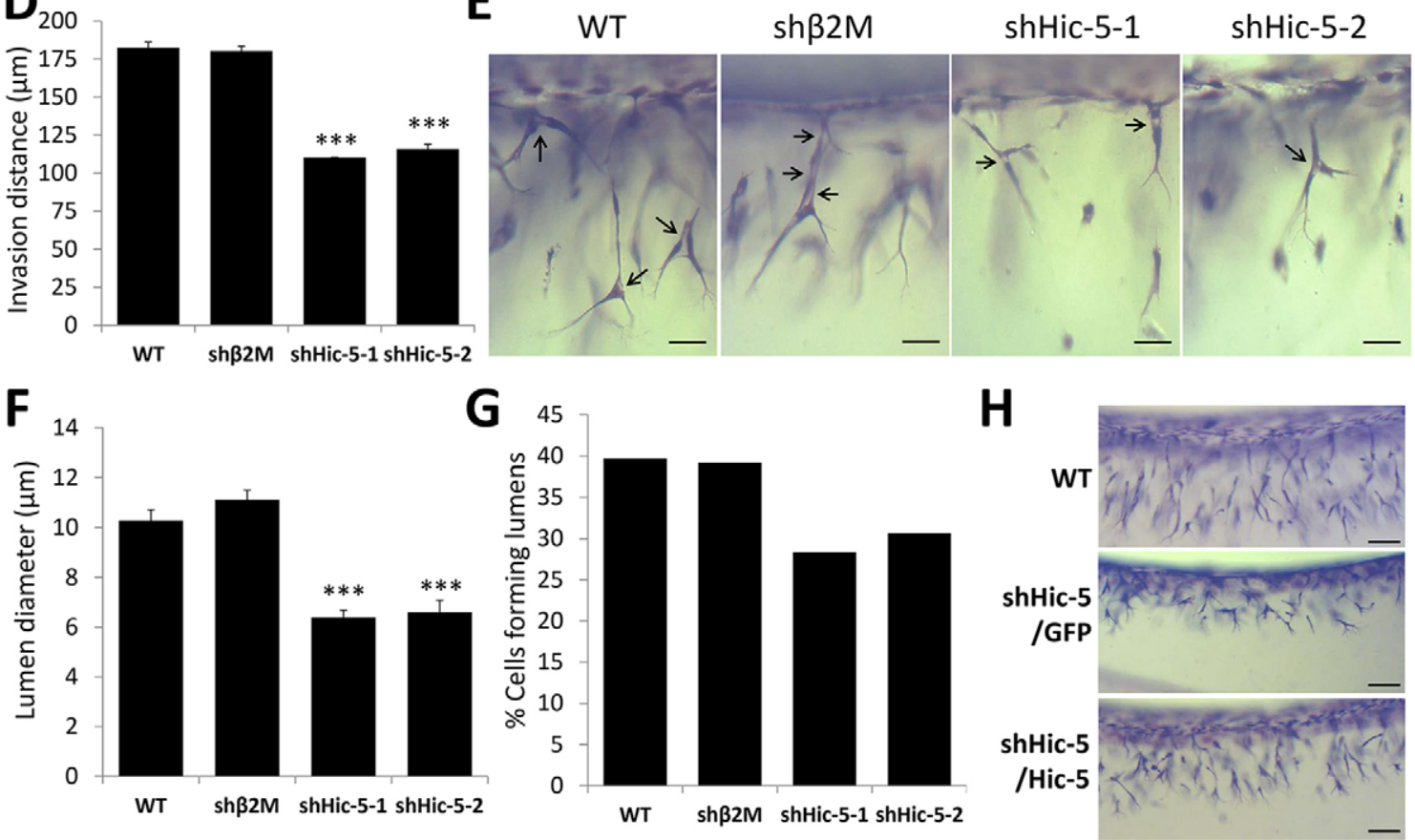

H
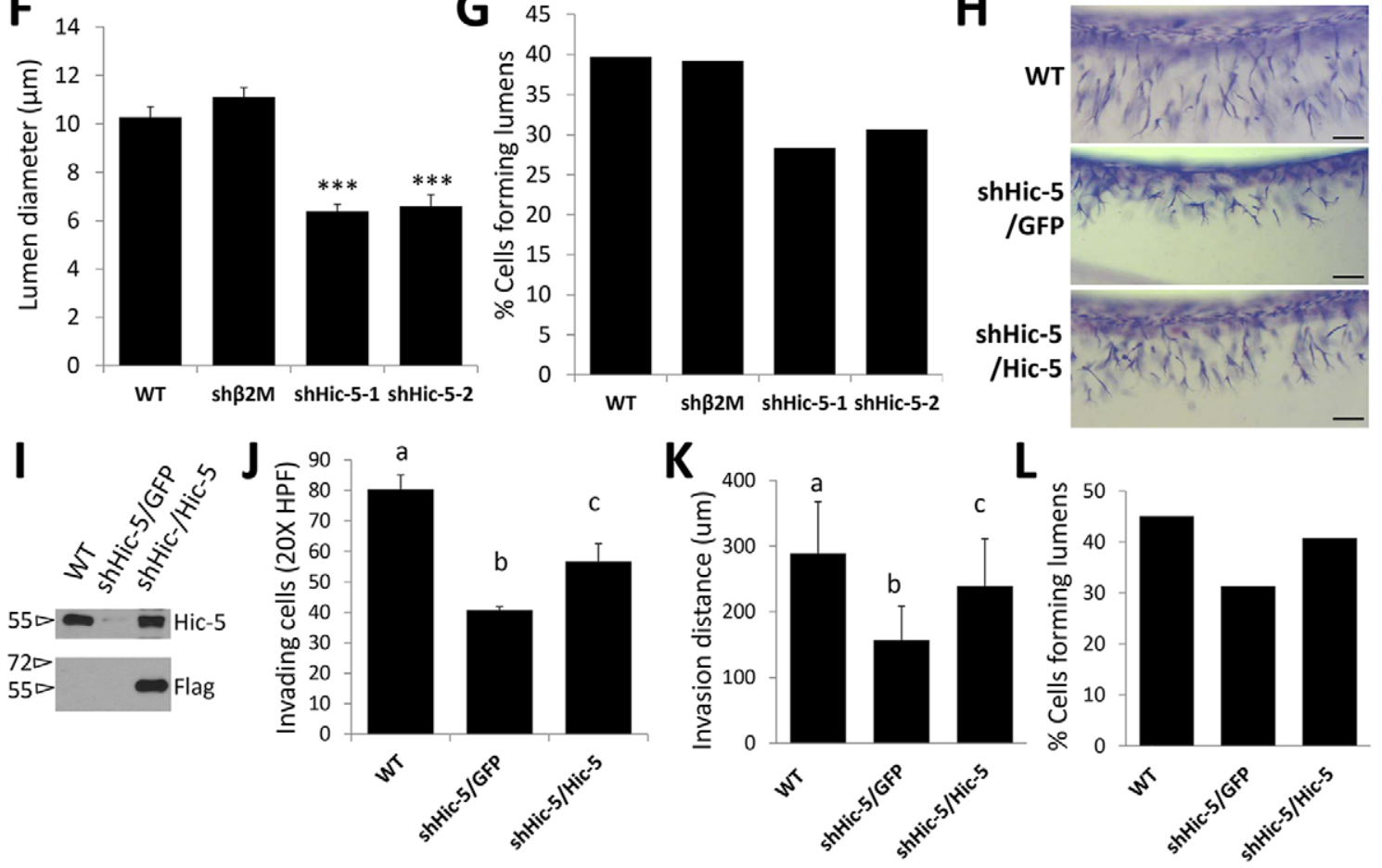

Fig. 1. Hic-5 silencing decreases S1P- and growth-factor-mediated endothelial cell invasion and lumen formation in 3D matrices. (A) Non-transduced endothelial cells (WT) and endothelial cells transduced with lentiviruses delivering shRNA directed against $\beta 2$-microglobulin (sh $\beta 2 \mathrm{M}$ ) or Hic-5 (shHic-5-1 and shHic-5-2) were tested in 3D invasion assays using S1P and growth factors. Invasion cultures were fixed at $20 \mathrm{~h}$ and imaged to illustrate the side view of invasion responses. Scale bars: $75 \mu \mathrm{m}$. (B) Cell lysates were analyzed by western blotting using Hic-5-, actin-, tubulin-, GAPDH-, and $\beta 2 \mathrm{M}-\mathrm{specific}$ antisera to verify $\beta 2 \mathrm{M}$ and Hic-5 protein suppression. Quantification of $(C)$ invasion density and $(D)$ invasion distance at $20 \mathrm{~h}$ of invasion. Data in $C$ represent the mean \pm s.d. number of invading cells per $1 \mathrm{~mm}^{2}$ field ( $n=3$ fields). Data in D represent the mean \pm s.e.m. length of structures. $\left(n>100\right.$ cells). ${ }^{* *} P<0.01 ;{ }^{* * *} P<0.001$ versus sh $\beta 2 \mathrm{M}$ (Student's $t$-test). A representative experiment is shown ( $n=4$ total). (E) Images of a side view of invading structures from non-transduced endothelial cells (WT) or endothelial cells transduced with lentiviruses delivering shRNA directed against $\beta 2 \mathrm{M}(\mathrm{sh} \beta 2 \mathrm{M}$ ) or Hic-5 (shHic-5-1, and -2 ). Arrows indicate lumens. Scale bars: $75 \mu \mathrm{m}$. Quantification of $(\mathrm{F})$ mean \pm s.e.m. lumen diameter (in microns) and $(\mathrm{G})$ mean percentage of cells forming lumens $\left(n>100\right.$ cells). ${ }^{* * *} P<0.001$ versus sh $\beta 2 \mathrm{M}$ (Student's $t$-test). Results in $\mathrm{E}-\mathrm{G}$ are from one representative experiment $(n=3)$. (H-L) A representative rescue experiment $(n=4$ total) quantified as in $\mathrm{D}$, $\mathrm{E}$ and F. (H) Images (side view) of the invasion responses of non-transduced endothelial cells (WT) or endothelial cells expressing Hic-5 shRNA and either GFP or Flag-tagged full-length Hic-5 rescue constructs. Scale bars: $100 \mu \mathrm{m}$. (I) Western blot from cell extracts probed with Hic-5 and Flag-specific antisera. Quantification of $(\mathrm{J})$ mean \pm s.d. invasion density, $(\mathrm{K})$ mean \pm s.d. invasion distance, and $(\mathrm{L})$ the mean percentage of cells forming lumens. Statistical analyses in $\mathrm{J}$ and $\mathrm{K}$ were performed using one-way ANOVA with Tukey's multiple comparisons test. Different letters indicate statistically significant differences with the following multiplicity adjusted $P$-values: (J) $P<0.0001$ for a versus b; $P<0.01$ for a versus c; $P<0.05$ for b versus $\mathrm{c}$; and (K) $P<0.0001$ for all comparisons. 
A
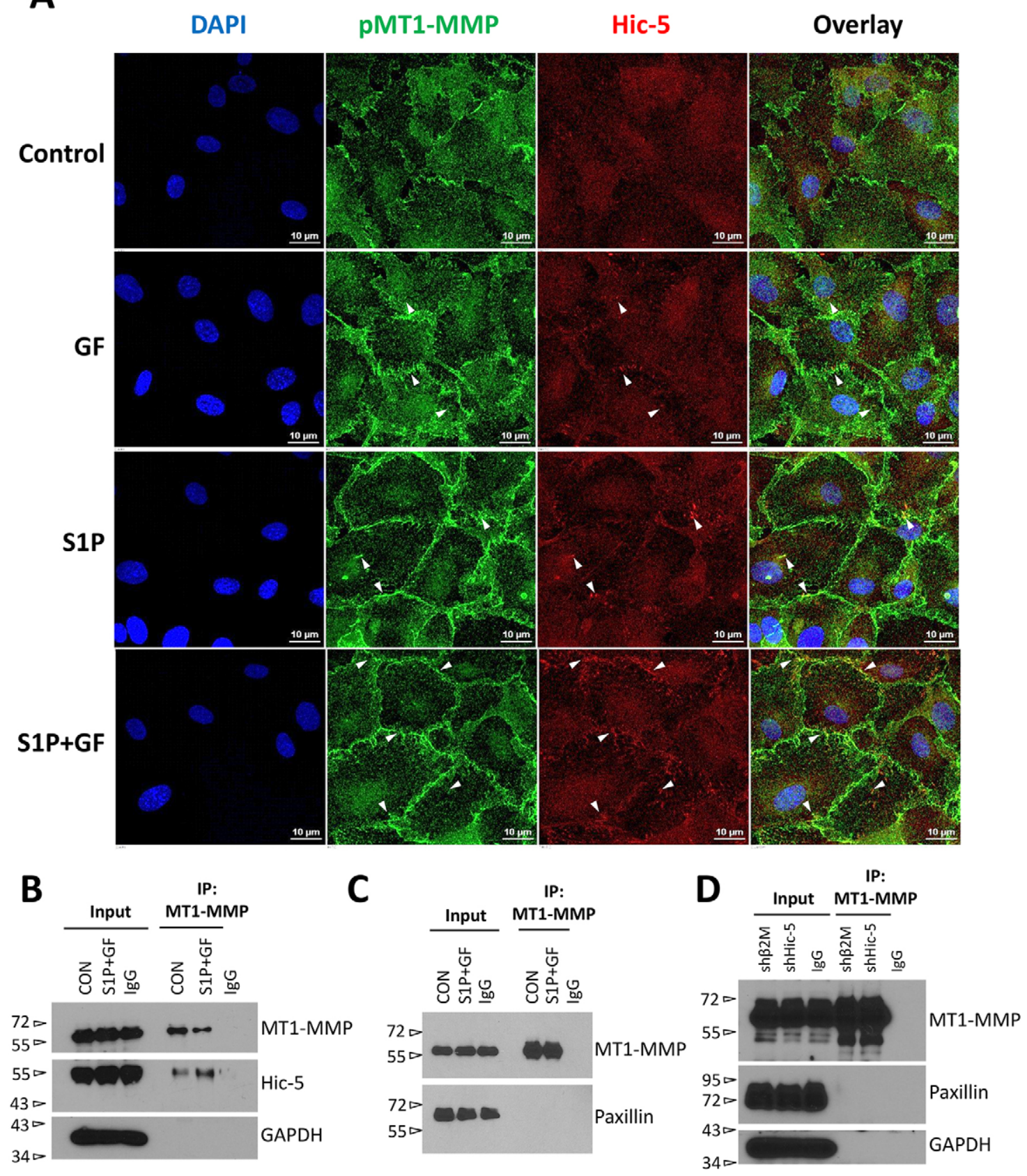

Fig. 2. Hic-5, but not paxillin, forms a complex with MT1-MMP in response to activation. (A) Endothelial cells seeded overnight on collagen-coated coverslips were serum starved for $2 \mathrm{~h}$ and left untreated (Control) or treated with $40 \mathrm{ng} / \mathrm{ml} \mathrm{VEGF} \mathrm{and} \mathrm{bFGF} \mathrm{(GF),} 1 \mu \mathrm{M} \mathrm{S1P}$ (S1P) or both (S1P+GF) for $1 \mathrm{~h}$. Cells were probed with antibodies directed against Hic-5 and phosphorylated MT1-MMP (pMT1-MMP Y573) followed by secondary antibodies conjugated to Alexa Fluor 594 and 488, respectively, and counterstained with DAPI (blue). Image stacks were collected using confocal microscopy. Arrowheads show areas of colocalization. Scale bars: $10 \mu \mathrm{m}$. (B,C) Endothelial cells cultured in 10-cm dishes were stimulated with nothing (CON) or $1 \mu \mathrm{M} \mathrm{S1P}+40 \mathrm{ng} / \mathrm{ml}$ growth factors (S1P +GF) for $1 \mathrm{~h}$. Immunoprecipitations (IP) were performed using polyclonal MT1-MMP antibodies or isotype (IgG) controls. Eluates were probed with (B) MT1-MMPGAPDH-, and Hic-5-specific antisera or (C) MT1-MMP- and paxillin-specific antisera in western blot analyses. (D) Immunoprecipitations with MT1-MMP-specific antibodies were performed with endothelial cells expressing shB2M or shHic-5 and treated with S1P+GF for $1 \mathrm{~h}$. Eluates were probed with MT1-MMP-, paxillinand GAPDH-specific antisera in western blot analyses. All experiments were performed four times, and a representative experiment is shown.

MMP constructs were co-transfected with Hic-5 (FL), $\Delta$ LIM4, $\Delta$ LIM3-4, $\Delta$ LIM2-4, $\Delta$ LIM2, $\Delta$ LIM3 or $\Delta$ LIM2-3 (Fig. 3A) in 293 cells. Lysates were incubated with MT1-MMP- specific or control (IgG) antisera, and eluates were probed with MT1-MMP- and Flagspecific antibodies. Western blot analyses revealed that there was a substantial amount of MT1-MMP association with full-length Hic-5 and $\triangle$ LIM4, but the association was slightly decreased with the
$\Delta$ LIM3-4, $\Delta$ LIM2 and $\Delta$ LIM3 constructs (Fig. 3C). MT1-MMP did not form a complex with the $\Delta$ LIM2-4 or $\Delta$ LIM2-3 constructs, supporting that the LIM2 and LIM3 domains within the C-terminus of Hic-5 are essential for formation of the complex with MT1-MMP. Based on results from these deletion constructs, we generated a Hic-5 construct containing only the C-terminal LIM2 and LIM3 domains (Hic-5 LIM2-3) to determine whether 
A
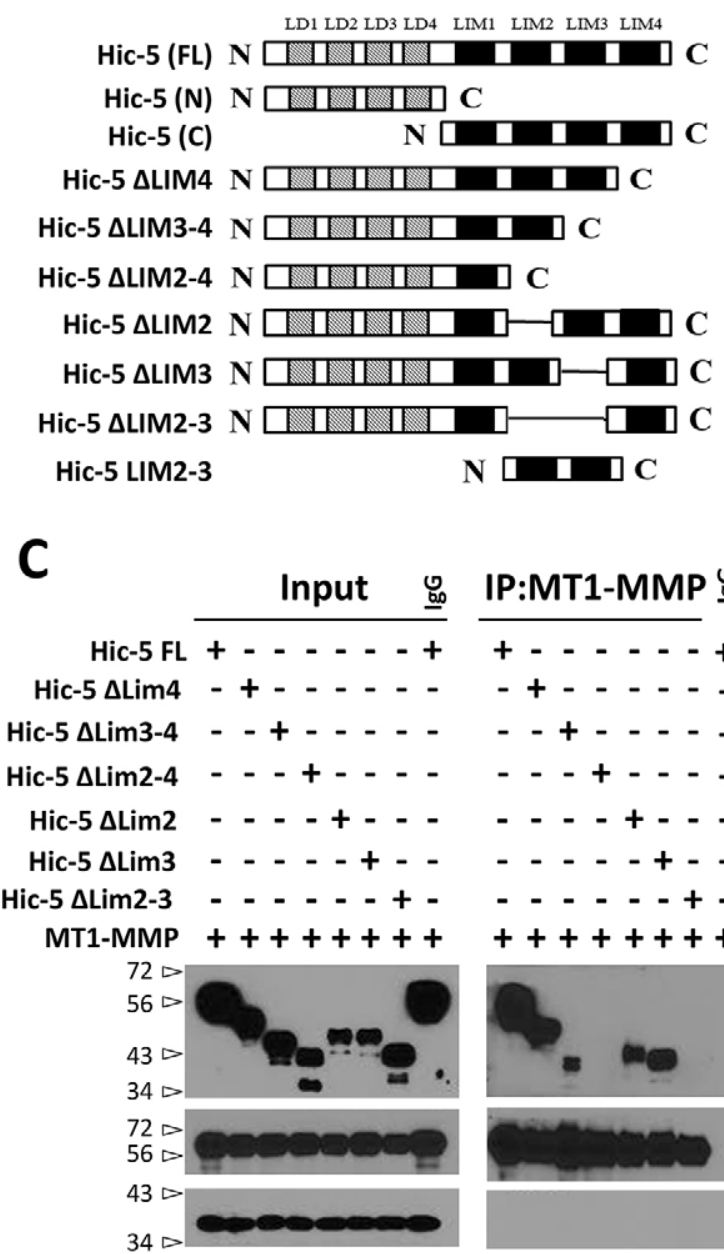

IP:MT1-MMP $\stackrel{0}{\stackrel{0}{0}}$

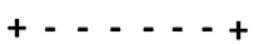

+ + - - -

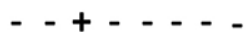

- - + - -

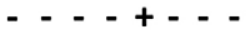

- - - - + -

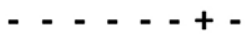

$++++++$

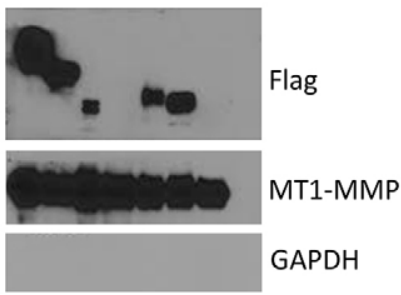

IP:

B
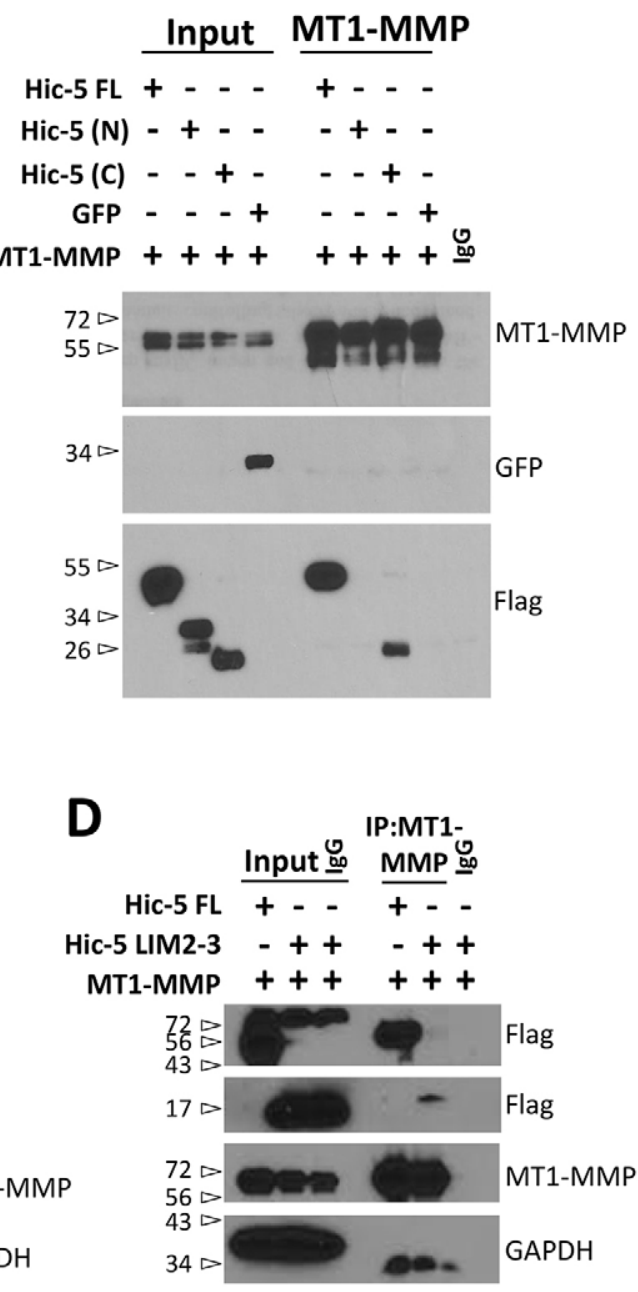

Fig. 3. The LIM2 and LIM3 domains of Hic-5 are required for MT1-MMP complex formation. (A) Schematic representation of Hic-5 expression constructs containing N-terminal Flag epitopes. (B) HEK-293 cells were co-transfected with MT1-MMP-His and Flag-tagged full-length (FL), N-terminal (N), or C-terminal (C) Hic-5 expression constructs, or a GFP control. Immunoprecipitation (IP) experiments were performed using polyclonal MT1-MMP antibodies or isotype (IgG) controls. Eluates were probed with MT1-MMP-, GFP- and Flag-specific antisera in western blot analyses. (C) HEK-293 cells were co-transfected with MT1-MMP-His and Flag-tagged Hic-5 FL, $\Delta$ LIM4, $\Delta$ LIM3-4, $\Delta$ LIM2-4, $\Delta$ LIM2, $\Delta$ LIM3 and $\Delta$ LIM2-3 expression constructs. Immunoprecipitation experiments were performed using polyclonal anti-MT1-MMP antibodies or isotype (IgG) controls. Eluates were probed with MT1-MMP-, Flag- and GAPDH-specific antisera in western blot analyses. (D) HEK-293 cells were co-transfected with MT1-MMP-His and Flag-tagged Hic-5 FL or Hic-5 LIM2-3 constructs. Immunoprecipitation experiments were performed as in B and C. Representative experiments are shown $(n=3)$.

expression of these domains was sufficient for it to form a complex with MT1-MMP. Western blot analyses demonstrated that MT1MMP formed a complex with both the full-length and LIM2-3 constructs (Fig. 3D), indicating that the LIM2 and LIM3 domains are both necessary and sufficient for Hic-5 to form a complex with MT1-MMP.

\section{Hic-5 and MT1-MMP colocalized in invading endothelial cells, and complex formation is enhanced by pro-angiogenic factors}

Fig. 2 supports that Hic-5 and MT1-MMP form a complex in activated endothelial cell monolayers. To examine the localization of these proteins in 3D sprouting structures, endothelial sprouts were stained with Hic-5- and pMT1-MMP-specific antisera. Although both Hic-5 and pMT1-MMP were distributed throughout the entire invading structure, we observed colocalization at punctate structures within extended fine processes at the periphery, as well as at the leading edge of sprouting structures (Fig. 4A; Fig. S4A). To determine whether complex formation between Hic-5 and MT1-MMP was upregulated during endothelial sprouting, immunoprecipitation experiments were performed with cultures treated with or without S1P and growth factors. Lysates were immunoprecipitated using MT1-MMP-specific antibodies and probed for MT1-MMP and Hic-5 by western blotting (Fig. 4B). Quantification of these experiments revealed a significant increase in Hic-5 and MT1-MMP complex formation with S1P and growth factor $(\mathrm{S} 1 \mathrm{P}+\mathrm{GF})$ stimulation in invading compared to non-invading (CON) endothelial cells (Fig. 4C). As a membrane-anchored metalloproteinase, MT1-MMP is well-suited to accomplish ECM proteolysis when present at the cell surface (Hiraoka et al., 1998; Murphy and Gavrilovic, 1999). To determine whether MT1-MMP and Hic-5 were localized within detergent-resistant membrane (DRM) fractions during invasion, 3D cultures were stimulated with or without S1P and growth factors and extracted with increasing concentrations of detergent prior to collection of the DRM fraction. In agreement with a previous study (Kwak et al., 2012), we observed 

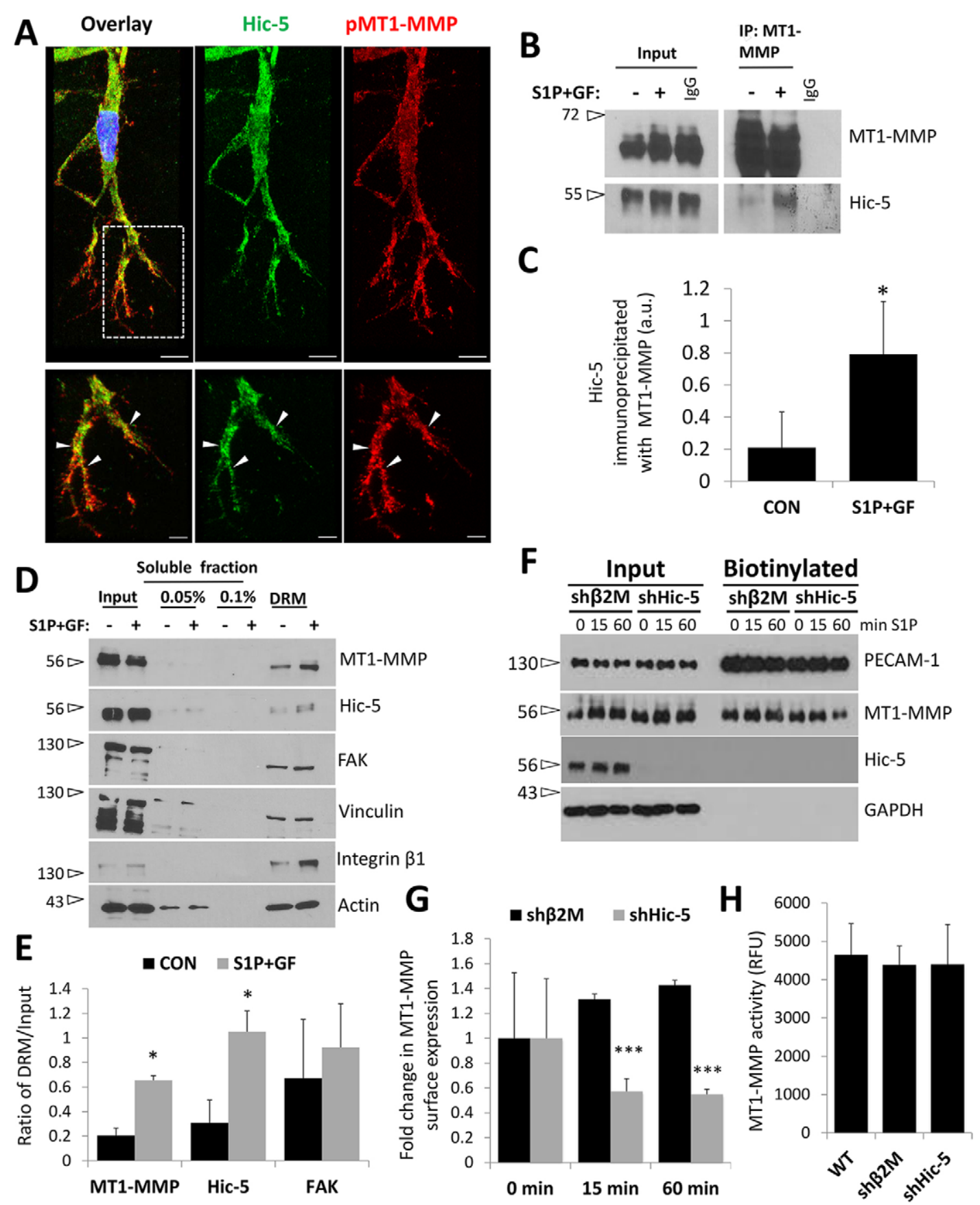

Fig. 4. Hic-5 and MT1-MMP interactions are enhanced during endothelial sprouting. (A) Frozen sections of endothelial sprouts in 3D collagen matrices were stained with anti-Hic-5 and anti-pMT1-MMP (Y573) antibodies followed by species-specific secondary antibodies conjugated to Alexa Fluor 488 and Alexa Fluor 594, respectively, and counterstained with DAPI (blue). Images were collected using confocal microscopy. The upper panel shows a full view of the sprout. The lower panel shows a magnified view of selected area marked above. Arrowheads indicate areas of overlap between Hic-5 and pMT1-MMP in the fine processes of the sprout. Scale bars: $5 \mu \mathrm{m}$ (upper panel); $2 \mu \mathrm{m}$ (lower panel). (B) 3D invasion cultures were established with no treatment (-) or with $1 \mu \mathrm{M} \mathrm{S1P}$ and $40 \mathrm{ng} / \mathrm{ml}$ growth factors $(G F,+)$ and allowed to invade collagen matrices for $4 \mathrm{~h}$ prior to detergent extraction. Immunoprecipitations (IP) were performed using polyclonal antibodies directed against MT1-MMP or isotype (IgG) controls. Eluates were probed for Hic-5 and MT1-MMP in western blot analyses. (C) Quantification of coprecipitated Hic- 5 normalized to the amount of starting material from western blots using Image J software. The results represent mean \pm s.d. in arbitrary units (a.u.) from three independent experiments. ${ }^{*} P<0.05$, compared to CON (Student's $t$-test). (D) DRM fractions were isolated from invading cultures without (-) or with $1 \mu \mathrm{M} \mathrm{S1P}$ and $40 \mathrm{ng} / \mathrm{ml}$ growth factors (+). Starting material lysates, soluble lysates, and DRM fractions were analyzed by performing western blotting with antibodies directed against MT1-MMP, Hic-5, FAK, vinculin, the $\beta 1$ integrin subunit and actin. (E) Quantification of MT1-MMP, Hic-5, and FAK levels in DRM normalized to the amount of starting material from western blots using ImageJ software. The results represent mean \pm s. $d$. in arbitrary units from three independent experiments. ${ }^{*} P<0.05$, compared to $\mathrm{CON}$ (Student's $t$-test). (F) Surface biotinylation experiments comparing surface labeling on endothelial cells expressing sh $\beta 2 \mathrm{M}$ and shHic-5. Cells were treated with S1P for 0-60 min, labeled and extracted. Biotinylated materials were captured with streptavidin-Sepharose and eluates were probed with antibodies directed against PECAM1, MT1-MMP, Hic-5 and GAPDH in western blot analyses. (G) Quantification of fold change in MT1-MMP surface expression normalized to PECAM1 loading controls from western blots of surface biotinylation. Data from three experiments were averaged to determine mean \pm s.d. values. (H) Quantification (mean \pm s.d.) of MT1-MMP activation at $6 \mathrm{~h}$ of invasion in wild-type (WT), sh $\beta 2 \mathrm{M}$ and shHic-5 cells. One representative experiment is shown $(n=3)$. 
successful upregulation of MT1-MMP in DRM fractions in invading endothelial cells upon S1P and growth factor treatment (Fig. 4D). We also observed increased levels of Hic-5 in DRM fractions of sprouting endothelial cells. Although vinculin levels remained unaffected by $\mathrm{S} 1 \mathrm{P}$ and growth factor treatment, the amount of the $\beta 1$ integrin subunit was also increased in DRM fractions of invading endothelial cells (Fig. 4D). Because Hic-5 and the $\beta 1$ integrin subunit were upregulated, we also analyzed the levels of FAK, which is known to be associated with integrins (Guan, 1997; Chen et al., 2000) and Hic-5 (Matsuya et al., 1998). Interestingly, levels of FAK and actin remained unchanged (Fig. 4D). Quantification of MT1-MMP, Hic-5 and FAK levels in DRM fractions revealed an increased presence of Hic-5 and MT1-MMP in DRM fractions of invading compared to non-invading cultures, but no significant change in FAK localization (Fig. 4E).

To determine whether Hic-5 regulates plasma membrane localization of MT1-MMP, surface biotinylation experiments were conducted with endothelial cells expressing shRNA directed against $\beta 2 \mathrm{M}(\mathrm{sh} \beta 2 \mathrm{M})$ or Hic-5 (shHic-5). Endothelial cells were treated with S1P, which is known to promote MT1MMP translocation to the plasma membrane (Nyalendo et al., 2007). Western blot analyses confirmed Hic-5 silencing and enrichment of surface biotinylated molecules (determined using anti-PECAM1 antibodies), and no contamination from cytoplasmic proteins (by using GAPDH controls) (Fig. 4F). Although S1P enhanced MT1-MMP on the surface in $\operatorname{sh} \beta 2 \mathrm{M}$ expressing cells, Hic-5 silencing resulted in a decrease in MT1-MMP compared to sh $32 \mathrm{M}$-expressing cells (Fig. 4G). Importantly, Hic-5 silencing did not alter MT1-MMP activity levels (Fig. 4H). Taken together, these findings demonstrate that complex formation between Hic-5 and MT1-MMP in sprouting endothelial cells is enhanced with $\mathrm{S} 1 \mathrm{P}$ and growth factor activation and occurs in DRM fractions. Furthermore, in the absence of Hic-5, MT1-MMP does not properly localize to the plasma membrane, explaining a decrease in endothelial cell sprout initiation and lumen formation.

\section{S1P mediated rapid translocation of Hic-5 to focal adhesions and enhanced MT1-MMP and FAK interactions}

Because Hic-5 is a known focal adhesion scaffold protein that forms a complex with FAK (Fujita et al., 1998), and the fine punctate staining pattern within 3D sections (Fig. 4A) appears to be similar to those reported for other focal adhesion proteins in 3D (Cukierman et al., 2001; Petroll and Ma, 2003), we further investigated whether Hic- 5 colocalized with activated FAK. Endothelial cells seeded on collagen-coated coverslips were treated with S1P and probed with antisera specific for Hic-5 and FAK phosphorylated at Y397 (pFAK Y397) (Fig. S3A). S1P increased Hic-5 and pFAK Y397 overlap, suggesting that Hic-5 translocated to focal adhesion sites. Similar results were seen with $\mathrm{S} 1 \mathrm{P}+\mathrm{GF}$ treatment (data not shown). Because $\mathrm{S} 1 \mathrm{P}+\mathrm{GF}$ also enhanced Hic-5 colocalization with total and pMT1MMP (Fig. 2), we further investigated whether S1P enhanced the interaction of MT1-MMP with FAK. Endothelial cells were treated without $(\mathrm{CON})$ or with $1 \mu \mathrm{M} \mathrm{S1P}$, and lysates were immunoprecipitated using FAK-specific or control (IgG) antisera. We observed increased FAK and MT1-MMP association following stimulation with S1P, while equivalent amounts of FAK were immunoprecipitated in both conditions (Fig. 5A). Quantification of these experiments revealed that $\mathrm{S} 1 \mathrm{P}$ treatment significantly increased the formation of complexes between FAK and MT1MMP (Fig. S3B).
Focal adhesions have been implicated as potential sites for MT1MMP-dependent matrix degradation in tumor cells, and the association between MT1-MMP and FAK is required for optimal MT1-MMP activity and matrix degradation at these sites (Takino et al., 2007; Wang and McNiven, 2012). Like other paxillin family members, Hic-5 functions as a focal adhesion scaffold that mediates multiple protein-protein interactions at adhesion sites through its LD and LIM domains (Dawid et al., 1995; Shibanuma et al., 2012). Our data suggest that a complex forms between MT1-MMP and Hic-5 in endothelial cells following activation with $\mathrm{S} 1 \mathrm{P}+\mathrm{GF}$ (Fig. 2), which is required to stimulate sprouting in endothelial cells (Fig. S2A). In addition, we observed that FAK and MT1-MMP formed a complex in invading endothelial cells (Fig. 5B). In agreement with the immunoprecipitation results, FAK and pMT1MMP colocalized in invading endothelial cells at various sites (Fig. 5C; Fig. S4B). Similarly, Hic-5 and pFAK also colocalized within sprouting structures (Fig. 5D; Fig. S4C). Hic-5 knockdown also decreased endothelial cell attachment to collagen in adhesion assays (Fig. S2D). These results suggest that Hic-5, FAK, and MT1MMP are all part of a complex that controls endothelial cell sprout outgrowth, and loss of Hic-5 results in reduced endothelial cell adhesion to the ECM. Therefore, to test whether Hic-5 was required to mediate MT1-MMP and FAK association, we performed MT1MMP immunoprecipitations in endothelial cells expressing shHic-5 and $\operatorname{sh} \beta 2 \mathrm{M}$. Decreased Hic- 5 expression resulted in a decrease in the amount of FAK associated with MT1-MMP (Fig. 5E). These data support that, by complexing with MT1-MMP, Hic-5 enhanced the association between MT1-MMP and FAK.

\section{Hic-5 mutants interfered with endothelial cell sprout initiation}

Because the LIM2 and LIM3 domains of Hic-5 were needed for Hic-5 to complex with MT1-MMP, we next determined whether Hic-5 mutants lacking the LIM2 and LIM3 domains interfered with endothelial cell sprouting by comparing endothelial cells expressing GFP to those expressing Hic-5 $\triangle$ LIM2-3. Images illustrating invasion responses are shown in Fig. 6A, where Hic-5 $\Delta$ LIM2-3 partially interfered with invasion responses. Expression of the Flagtagged $\Delta$ LIM2-3 construct was confirmed by western blotting (Fig. 6B). GFP expression was confirmed visually (data not shown). Quantification of invasion responses revealed that expression of Hic-5 $\Delta$ LIM2-3 significantly reduced the number of invading cells (Fig. 6C). Although a trend towards decreased lumen diameter, the percentage of cells that formed lumens, and invasion distance was observed (Fig. 6D-F), no significant differences were detected, suggesting that the Hic-5 $\Delta$ LIM2-3 mutant was only able to interfere with sprouting initiation, perhaps due to the presence of endogenous Hic-5 in these experiments.

\section{Hic-5, MT1-MMP and FAK are co-expressed in developing blood vessels during porcine pregnancy in vivo}

Our results demonstrate that Hic-5 might act as a molecular scaffold to mediate complex formation between MT1-MMP and FAK. To determine whether these molecules are present and colocalize during angiogenesis in vivo, we analyzed an early stage of porcine pregnancy. Successful implantation results in close apposition of fetal (chorionic) and maternal (luminal) epithelium that is supported by a well-defined sub-epithelial layer of angiogenic vessels that facilitate nutrient exchange between maternal and fetal tissues (Senger, 2011). To determine whether Hic-5, MT1-MMP and FAK were expressed in developing endothelium, we co-stained with VEcadherin to label areas of subepithelial angiogenesis (Fig. 7A-C). 

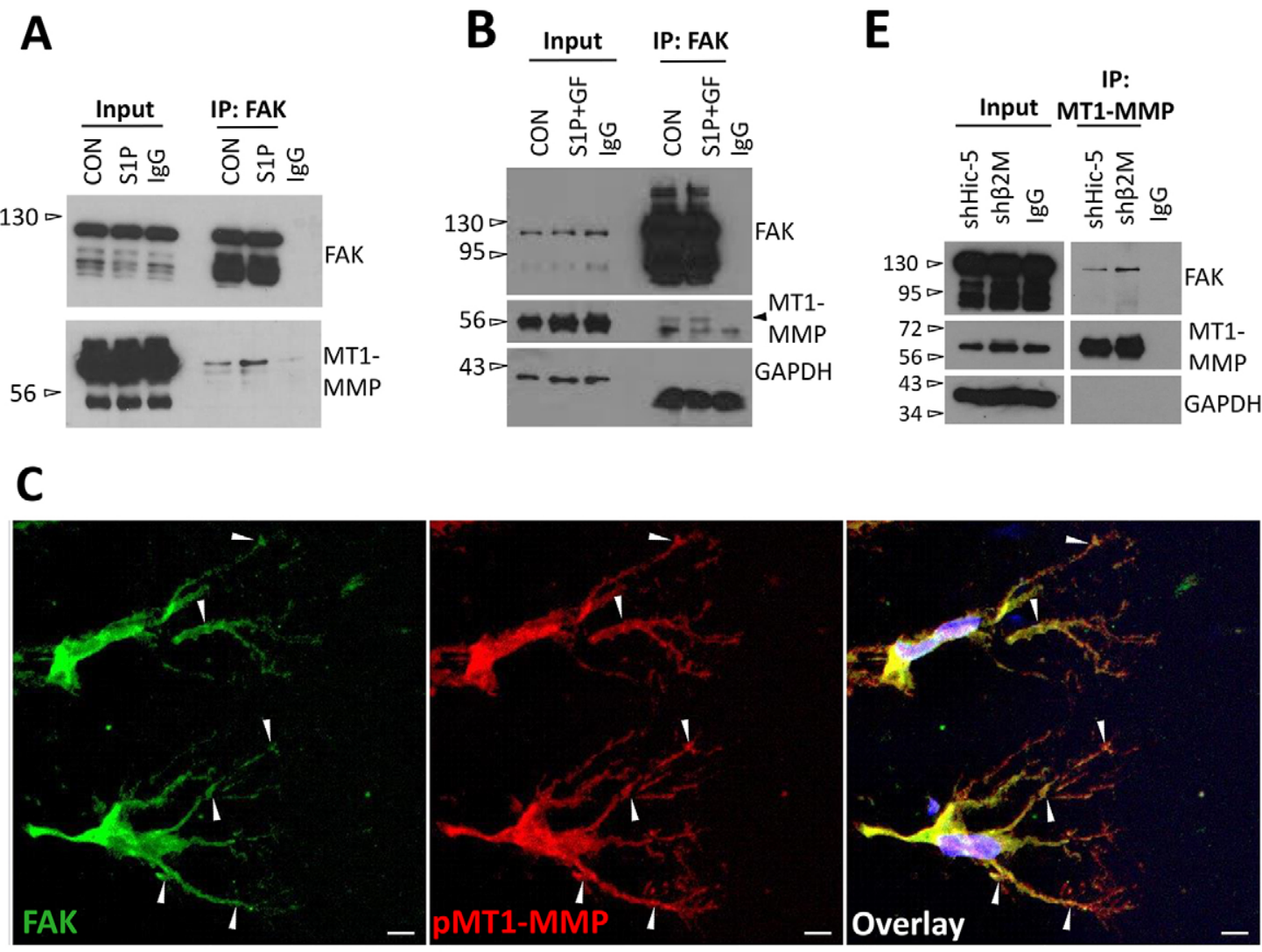

D
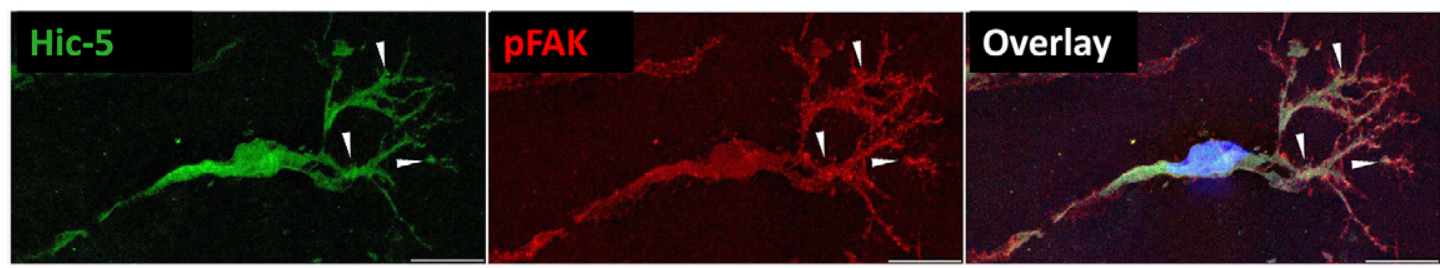

Fig. 5. MT1-MMP and FAK interactions occur during sprouting and are mediated in part through Hic-5. (A) Endothelial cells cultured in 10-cm dishes were stimulated with nothing (CON) or $1 \mu \mathrm{M} \mathrm{S1P}$ for $1 \mathrm{~h}$. Immunoprecipitations (IP) were performed using monoclonal FAK antibodies or isotype (IgG) controls. Eluates were probed with MT1-MMP- and FAK-specific antisera in western blot analyses. (B) Invasion cultures were established with no treatment (CON) or with S1P and growth factor (S1P+GF) and allowed to invade collagen matrices for $6 \mathrm{~h}$ prior to performing immunoprecipitations with polyclonal antibodies directed to $\mathrm{FAK}$ or isotype (IgG) controls. Eluates were probed for FAK, MT1-MMP and GAPDH in western blot analyses. Frozen sections of endothelial sprouts in 3D collagen matrices were stained with (C) anti-FAK and anti-pMT1-MMP (Y573) antibodies or (D) Hic-5 and pFAK (Y397) antisera. Arrowheads show areas of colocalization. Scale bars: $10 \mu \mathrm{m}$. (E) Endothelial cell monolayers in 10-cm dishes expressing sh $\beta 2 \mathrm{M}$ and shHic-5 were treated with S1P+GF for $1 \mathrm{~h}$ prior to detergent extraction. Immunoprecipitations were performed using polyclonal antibodies directed against MT1-MMP or isotype (IgG) controls. Eluates were probed for FAK, MT1-MMP and GAPDH in western blot analyses. A representative experiment is shown ( $n=3$ total).

We observed that VE-cadherin-positive endothelium expressed Hic-5, MT1-MMP and FAK, indicating that these proteins were expressed in angiogenic blood vessels during pregnancy. Furthermore, we observed colocalization of pMT1-MMP (Y573) and Hic-5 (Fig. 7D), pMT1-MMP (Y573) and FAK (Fig. 7E), and pFAK (Y397) and Hic-5 (Fig. 7F) in sub-epithelial blood vessels. Taken together, these data support that Hic-5, MT1-MMP and FAK are present in angiogenic endothelium and, thus, are available to participate in angiogenic events during pregnancy in vivo.

\section{DISCUSSION}

This study reveals a new regulatory role for the focal adhesion scaffold protein Hic-5 during sprouting angiogenesis in 3D collagen matrices. Our results suggest that Hic-5 formed a complex with MT1-MMP, regulated MT1-MMP plasma membrane translocation, and mediated FAK and MT1-MMP interactions to promote endothelial invasion of 3D collagen matrices. These data support the possibility that Hic-5, through interactions with MT1-MMP and FAK, coordinates matrix proteolysis events with cell migration during endothelial invasion.

The complex formation between Hic-5 and MT1-MMP has important implications for cell invasion in 3D. MT1-MMP is a membrane-anchored metalloproteinase that cleaves matrix proteins in close proximity to the cell surface, thus maintaining overall matrix integrity to support invading structures (Werb, 1997; Hiraoka et al., 1998; Murphy and Gavrilovic, 1999; Hotary et al., 2000). MT1-MMP has been shown to direct endothelial sprouting and lumen formation, as well as tumor cell invasion (Hiraoka et al., 1998; Yana and Seiki, 2003; Chun et al., 2004; Stratman et al., 2009). Following translation, the pro-domain of MT1-MMP is 
A

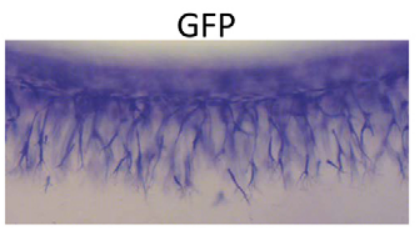

Hic-5 $\Delta$ LIM2-3

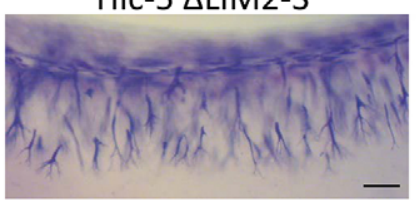

D

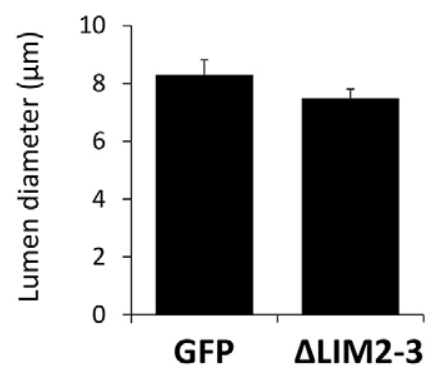

B

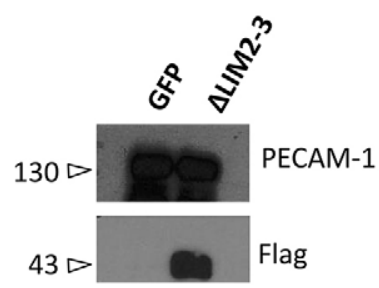

E

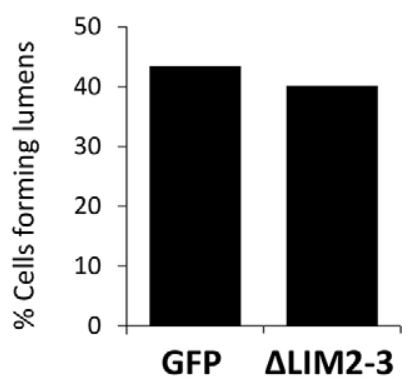

C

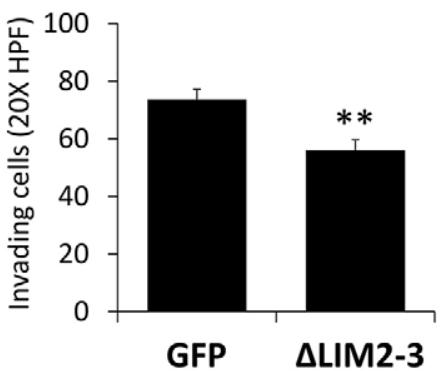

F

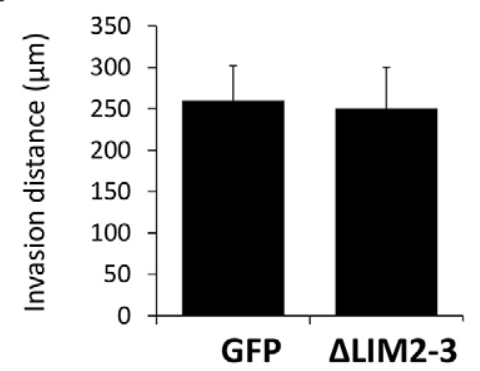

Fig. 6. Expression of Hic-5 mutant constructs interferes with endothelial invasion. (A) Images (side view) of invasion responses in endothelial cells expressing GFP or the Hic-5 $\Delta$ LIM2-3 construct (24 h). Scale bar: $100 \mu \mathrm{m}$. (B) Western blot of cell lysates from A probed with Flag- and PECAM1-specific antisera in western blot analyses. GFP expression was confirmed visually (not shown). Quantification of (C) mean \pm s.d. invasion responses ( $n=3$ fields), (D) mean \pm s.e.m. lumen diameter ( $n \geq 53$ cells), (E) the mean percentage of cells forming lumens ( $n \geq 122$ cells), and $(F)$ the mean \pm s.e.m. invasion distance ( $n \geq 100$ cells).

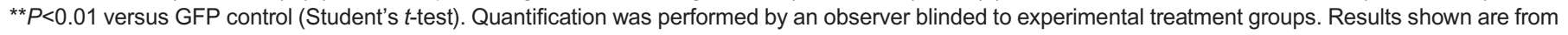
one representative experiment $(n=3)$.

removed by furins, generating the active form of MT1-MMP (Yana and Weiss, 2000; Osenkowski et al., 2004). Once activated, MT1MMP translocates to the surface where it can cleave ECM proteins (Remacle et al., 2006). In addition, active MT1-MMP undergoes endocytosis and can be recycled from the cell surface to early and late endosomes (Remacle et al., 2003). The activity assays performed were conducted on total cell lysates and cannot distinguish between surface-associated and internalized MT1MMP, explaining why no gross differences were observed in MT1-MMP activity. For this reason, we performed surface biotinylation assays, which demonstrated decreased surface expression of MT1-MMP that correlated with decreased invasion responses in the absence of Hic-5. Thus, Hic-5 silencing did not alter overall MT1-MMP activity levels, supporting that surface localization of MT1-MMP, rather than overall activity, is a key indicator of invasive behavior (Hotary et al., 2000; Yana and Weiss, 2000; Uekita et al., 2001; Egeblad and Werb, 2002; Li et al., 2008; Kwak et al., 2012). The observation that Hic-5 depletion decreased S1P-mediated MT1-MMP plasma membrane translocation in surface biotinylation assays, suggests that Hic-5 can function as a molecular adaptor to transport activated MT1-MMP to the plasma membrane for successful sprouting responses. Another possibility, not ruled out here, is that the absence of Hic-5 might accelerate internalization of MT1-MMP. Nevertheless, we observed colocalization of Hic-5 with pMT1-MMP in 3D endothelial sprouts, and MT1-MMP and Hic-5 levels were enhanced in DRM fractions of invading endothelial cells, indicative of membrane localization during endothelial sprouting responses. We further observed an increase in Hic-5 and MT1-MMP interactions when comparing invading to non-invading endothelial cells, suggesting that the Hic-5 and MT1-MMP complex is associated with the membrane compartment and contributes, at least in part, to successful endothelial sprouting responses in 3D matrices.

Our findings show that FAK is part of the Hic-5 and MT1-MMP complex that assembles in response to pro-angiogenic factors. These molecules colocalized in angiogenic vessels in vivo, and displayed a punctate staining pattern in invading endothelial cells in vitro, similar to other focal adhesion proteins in 3D (Cukierman et al., 2001; Petroll and Ma, 2003). Because Hic-5 is known to function as an adaptor protein, we tested whether Hic-5 might facilitate MT1-MMP and FAK interactions. We found that loss of Hic-5 decreased the formation of the complex between MT1-MMP and FAK, supporting that Hic-5 bridges MT1-MMP and FAK. Hic-5 is well-suited for this role as an adaptor protein given that the N-terminal LD domain of Hic-5 binds FAK (Fujita et al., 1998), and the C-terminal LIM2 and LIM3 domains are required for interaction with MT1-MMP (shown here). Hic-5 is known to localize to integrin-mediated sites of adhesion, where it coordinates cell migration in 2D (Brown and Turner, 2004; Tumbarello and Turner, 2007; Deakin and Turner, 2008). The placement of Hic-5 in close proximity to the plasma membrane and in detergent-resistant fractions (shown here) renders Hic-5 perfectly poised to participate in a complex with both MT1-MMP and FAK.

Collectively, these results suggest that Hic-5 participates in assembly of a complex at the endothelial cell surface that is needed for motility and matrix proteolysis during invasion. Others have shown that Hic-5 silencing decreases endothelial cell migration ( $\mathrm{Wu}$ et al., 2005a; Avraamides et al., 2007), and we find that depletion of Hic-5 results in reduced sprout initiation and decreased invasion distance. In MDA-MB-253 cells, Hic-5 depletion decreases the 

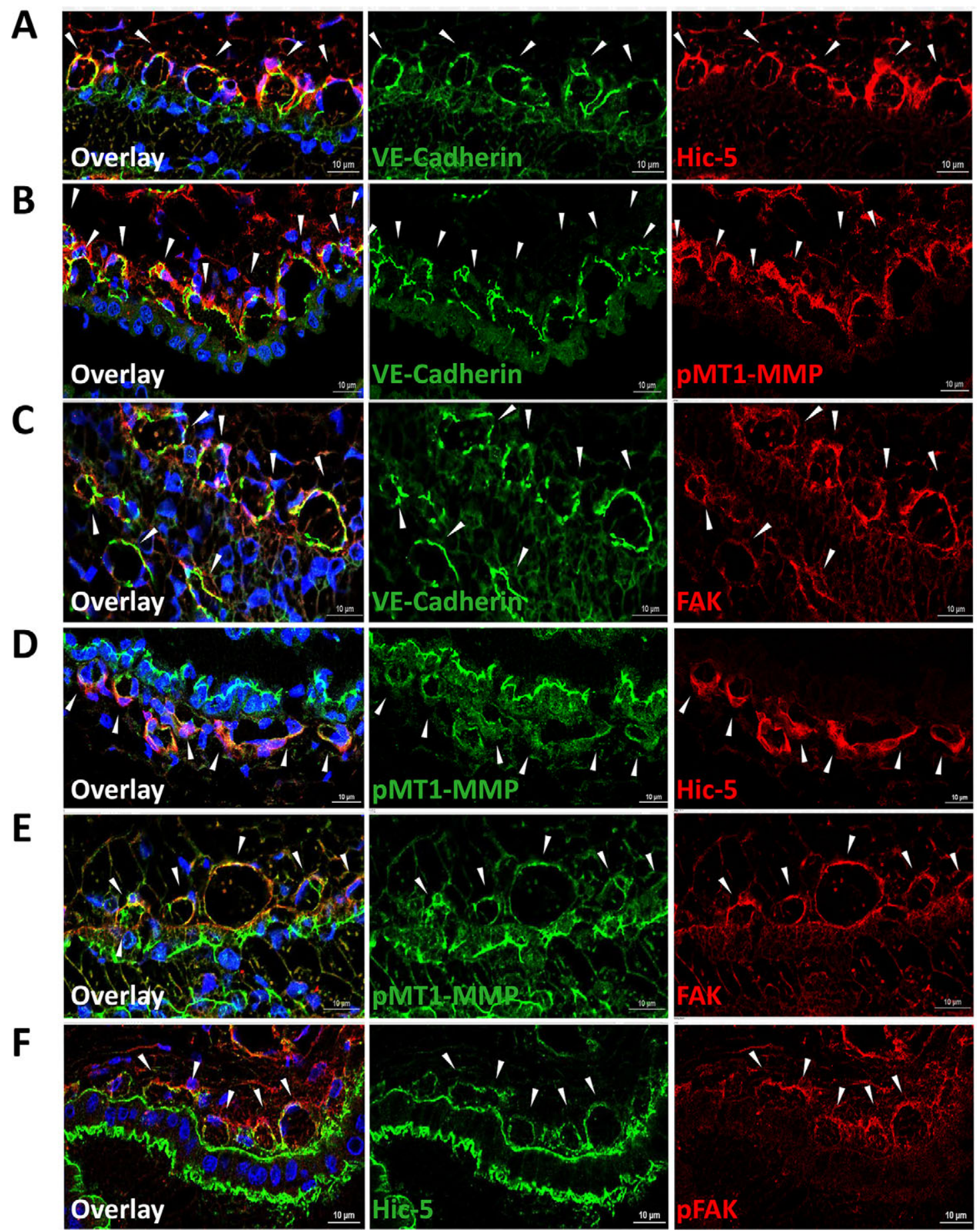

Fig. 7. Hic-5, MT1-MMP and FAK are co-expressed in developing blood vessels during porcine pregnancy in vivo. Frozen sections of porcine uterus at day 24 of pregnancy were fixed in methanol and probed with the antibodies indicated in each panel. Panels in the left column show overlay images from middle and right columns, as well as DAPI stain (blue). The indicated antibodies were detected with secondary antibodies conjugated to Alexa Fluor 488 (middle panels) and Alexa Fluor 594 (right panels). Image stacks were collected using confocal microscopy. Arrowheads indicate areas of overlap in capillary structures. Scale bars: $10 \mu \mathrm{m}$. Representative images are shown from a single animal of three analyzed in total.

number of vinculin-positive focal adhesions and motility in 3D (Deakin and Turner, 2011). Although a specific proteinase was not identified, Hic-5 depletion has been connected to decreased matrix degradation and filopodia formation (Pignatelli et al., 2012). Wang and McNiven have previously implicated focal adhesions as sites for MT1-MMP-dependent matrix degradation in tumor cells (Wang and McNiven, 2012), and others have suggested the importance of MT1-MMP and focal adhesion crosstalk during cell migration
(Wu et al., 2005b; Takino et al., 2006, 2007; Gingras et al., 2008). Importantly, expression of the Hic-5 $\Delta$ LIM2-3 mutants that did not interact with MT1-MMP partially reduced invasion, despite the presence of endogenous Hic-5. The LIM2 and LIM3 domains of paxillin, which are closely related to Hic-5, target paxillin to focal adhesions in fibroblasts (Brown et al., 1996). Collectively, the observations that the LIM2 and LIM3 domains in Hic-5 are needed to complex with MT1-MMP, and the ability of Hic-5 LIM2-3 
mutants to interfere with endothelial cell sprout initiation, support the idea that coupling MT1-MMP (which facilitates matrix proteolysis) to FAK (which provides cell traction) is conducive for invasion into 3D matrices. Although we cannot detect paxillin as part of a complex with MT1-MMP, this is not completely unexpected because paxillin and Hic- 5 have been demonstrated to play distinct roles in various systems (Kim-Kaneyama et al., 2005; Deakin et al., 2012). Taken together, the data presented here reinforce that, by recruiting a complex containing MT1-MMP and FAK, Hic-5 contributes to endothelial cell invasion in 3D collagen matrices.

Consideration of previous evidence suggests that Hic-5, MT1MMP and FAK are part of a larger signaling complex that assembles in response to endothelial cell activation by pro-angiogenic factors. Previously, p130Cas (also known as BCAR1) has been shown to facilitate the interaction between MT1-MMP and FAK in tumor cells (Wang and McNiven, 2012). S1P enhances the interaction between p130Cas and pMT1-MMP Y573 in endothelial cells, although this interaction is predicted to be indirect because the cytoplasmic tail of MT1-MMP lacks binding motifs for SH2 or SH3 domains to facilitate interactions with tyrosine-phosphorylated proteins (Gingras et al., 2008). The present study suggests that pro-angiogenic factors enhance the formation of the complex between Hic-5 and MT1MMP. Thus, it is tempting to speculate that S1P stimulation enhances interactions between Hic-5 and p130Cas in endothelial cells. Other potential interacting partners for Hic-5 include vimentin, which complexes with p130Cas (Wang et al., 2007), MT1-MMP (Kwak et al., 2012), caveolin (Labrecque et al., 2004), the $\alpha 2 \beta 1$ integrin (Sacharidou et al., 2010) and RACK1, which itself complexes with vimentin and FAK in invading endothelial cells (Dave et al., 2013). Taken together, these data suggest that Hic-5 might be a part of a larger signaling network involving other key molecules, whose assembly is initiated with endothelial cell activation. Additional studies will be necessary to definitively determine these interactions.

The data presented here add to a growing list of reports indicating that a link between matrix proteolysis and cell motility must be established for successful cell invasion in 3D. Our results indicate that Hic- 5 contributes to these events in endothelial cells by bridging MT1-MMP and FAK. FAK is a well-known regulator of angiogenesis (Peng et al., 2004; Shen et al., 2005), as is MT1-MMP (Zhou et al., 2000; Chun et al., 2004; Stratman et al., 2009). Thus, defining molecular events that coordinate and regulate the activities of these key proteins will further refine our understanding of new blood vessel growth.

\section{MATERIALS AND METHODS}

\section{Endothelial cell culture and invasion}

Certified single-donor human umbilical vein endothelial cells (HUVECs; Lonza, Allendale, NJ) at passages 3-6 were cultured as described previously (Bayless et al., 2009). 3D invasion experiments were established using $2.5 \mathrm{mg} / \mathrm{ml}$ collagen matrices containing $1 \mu \mathrm{M} \mathrm{S} 1 \mathrm{P}$ (Sigma, St Louis, MO). Confluent endothelial cell monolayers were seeded in M199 with $40 \mathrm{ng} / \mathrm{ml}$ VEGF and bFGF (R\&D Systems, Minneapolis, MN).

\section{Imaging and quantification of invasion density, distance and lumen size}

Side views of invading endothelial cells were captured using an Olympus CKX41 microscope equipped with a Q color 3 camera (Shinjuku, Tokyo, Japan). Invasion densities were quantified using at least three random fields to manually count the number of invading cells per field. For quantification of invasion distance, side-view cultures at $4 \times$ magnification were used to measure the distance the tip of each invading sprout traveled from the original monolayer using Image-Pro Analyzer 7.0. Approximately 100 cells per treatment group were included in all analyses. For quantification of lumen size, side-view images of cultures at $20 \times$ magnification were analyzed using Image-Pro Analyzer 7.0. At least 100 structures per treatment group were included in each analysis.

\section{Hic-5 and MT1-MMP cloning}

Flag-tagged Hic-5 constructs were generated in the pFlag-CMV2 vector (Sigma). HUVEC RNA was isolated using an RNeasy MiniKit (Qiagen, Valencia, CA), and cDNA was generated with the SuperScript III FirstStrand Synthesis System (Invitrogen, Grand Island, NY) using $1 \mu \mathrm{g}$ of RNA. A PCR reaction was performed using $3 \mu \mathrm{l}$ of cDNA template with AccuPrime Taq HIFI Polymerase (2.5 units, Invitrogen) in a 50- $\mu$ l reaction volume. The Hic-5 amplicons were cleaned with the Qiagen QIAquick PCR Purification Kit, digested with HindIII and $X b a \mathrm{I}$ (NEB, Ipswich, MA) along with the $\mathrm{pFlag}-\mathrm{CMV} 2$ vector at $37^{\circ} \mathrm{C}$ overnight, gel purified with the Qiagen QIAquick Gel Extraction Kit, and ligated overnight at $14^{\circ} \mathrm{C}$. Primers used for this study were: Flag-FL-Hic-5, 5'-AGCAAAGCTTGAGGACCTGGATGCCCTG-3' and 5'-AGCATCTAGATCAGCCGAAGAGCTTCAGG3'; Flag-N-Hic-5, 5'-AGCAAAGCTTGAGGACCTGGATGCCCTG-3' and 5'-AGCATCTAGACCGGCGGCTGAGGTCGGAC-3'; Flag-C-Hic5, 5'-AGCAAAGCTTGGT-GTTCCCACCCAGGCC-3' and 5'-AGCATCTAGATCAGCCGAAGAGCTTCAGG-3'; Flag- $\Delta$ LIM4, 5'-AGCAAAGCTTGAGGACCTGGATGCCCTG-3' and 5'-AGCATCTAGATCAGTGGAAGTGGTTCTCGCAC-3'; Flag- - LIM3-4, 5'-AGCAAAGCTTGAGGACCTGGATGCCCTG-3' and 5'-AGCATCTAGATCAGAAGTCCCGGCGGCAGTAG-3'; Flag- $\triangle$ LIM2-4, 5'-AGCAAAGCTTGAGGACCTGGATGCCCTG-3' and 5'-AGCATCTAGATCAAAAGTAGCACTCGGGGCAG-3'; and Flag-LIM2-3 Hic-5, 5'-AGCAAAGCTTTTCTCGCCAAGATGTGGC-3' and 5'-AGCATCTAGATCAGTGGAAGTGGTTCTCGCAC-3'. For deletion constructs, a PCR was performed using Flag fulllength Hic-5 template with AccuPrime Taq HIFI Polymerase (Invitrogen), and $0.4 \mu \mathrm{M}$ of forward and reverse primers containing a PacI (NEB) restriction site in a $50-\mu 1$ reaction volume. PCR products were treated with $1 \mu \mathrm{L} p n \mathrm{I}$ (NEB) for $1 \mathrm{~h}$ at $37^{\circ} \mathrm{C}$, cleaned with the Qiagen QIAquick PCR purification kit, digested with $P a c$ I, gel purified using Qiagen QIAquick Gel Extraction Kit, and ligated overnight at $14^{\circ} \mathrm{C}$. Primers used were: Flag $\triangle$ LIM2, 5'-CGCTTCTCGCCAAGATTAATTAAGTGTGGCTTCTGCAAC-3' and 5'-GGCGAACAGCTGCAGTTAATTAAGAAGTCCCGG-CGGCAG-3'; Flag-ALIM3, 5'-CTGTTCGCCCCGCGCTTAATTAAGTGCCAG-GGCTGCCAG-3' and 5'-CGAGCCGCGTCGTGCTTAATTAAGTGGAAGTGGTTCTC-3'; and Flag- $\triangle$ LIM 2-3, 5'-GAGAACCACTTCCACTTAATTAAGGCACGACGCGGCTCG-3' and 5'-GTTGCAGAAGCCACATTAATTAAGTCTTGGCGAGAAGCG-3'. Positive clones were verified by sequencing and cloned into the pENTR4 vector; inserts, which were sequence verified for full-length Hic-5, $\Delta$ LIM2 Hic-5, $\Delta$ LIM3 Hic-5, and $\triangle$ LIM2-3 Hic-5, in the pFlag-CMV2 vector were used as a template for amplification with primers 5'-AGCAGGATCCGCCACCATGGACTACAAAGACGATGAC-3' and 5'-AGCACTCGAGTCAGCCGAAGAGCTTCAGG $-3^{\prime}$. The amplicons were cleaned and digested with BamH1 and $X h o 1$ along with the pENTR 4 vector at $37^{\circ} \mathrm{C}$ overnight. Positive clones were verified by sequencing before being recombined with pLenti6/V5-DEST vector. Rescue constructs for full-length Hic-5 were amplified from full-length Hic-5 respectively, in the lenti backbone with the primers 5'-GTGAGCTAGATCGCTTGCTCCA-GGAACTCAATGCCACTCAG-3' and 5'-CTGAGTGGCATTGAGTTCCTGGAGCAAGCG-ATCTAGCTCAC-3' using the QuikChange Lightning Site-Directed Mutagenesis Kit (Stratgene) as per the manufacturer's instructions.

Human MT1-MMP was amplified from HUVEC cDNA using primers 5'-GATCGAATTCGCCACCATGTCTCCCGCCCCAAG-3' and 5'-CATGAAGCTTGACCTTGTCCAGCAGGG-3' and digested using EcoRI and HindIII restriction enzymes. Digested amplicons were ligated into the pET28b vector (Novagen), generating a C-terminal $6 \times$ His tag. Positive clones were verified by sequencing. MT1-MMP-His was amplified from the pET $28 \mathrm{~b}$ vector using primers 5'-CTAGGGTACCGCCACCATGTCTCCCGCCCCAAG-3' and 5'-GTACGATATCCTAGTGGTGGTGGTGGTGGTGGTGGTGCTCGAG- $3^{\prime}$, creating an $8 \times$ His tag. Amplicons were digested using $A c c 65 \mathrm{I}$ and EcoRV restriction enzymes and ligated into the pENTR4 vector. Positive clones were tested by sequencing and 
subsequently recombined with the pLenti6/V5-DEST vector in Stbl3 cells (Invitrogen). Clones were verified by sequencing.

\section{Endothelial cell transduction using lentiviral vectors}

Short hairpin RNA (shRNA) backbone vectors specific for Hic-5 (\#SHCL NG-NM015927; shHic-5-1 TRCN0000281350; shHic-5-2 TRCN000028 1352) and $\beta 2 \mathrm{M}$ (\#SHCLNG-NM004048 TRCN0000057254) were purchased from Sigma-Aldrich. Lentiviral particles were generated by transfecting $1.25 \mu \mathrm{g}$ of backbone shRNA plasmid with $3.75 \mu \mathrm{g}$ of VIRAPOWER packaging mix (Invitrogen) into confluent HEK-293 cells (Invitrogen) using Lipofectamine 2000 (Invitrogen) in T25 flasks. Viral supernatants were harvested at $60 \mathrm{~h}$, centrifuged at $1000 \mathrm{~g}$ for $10 \mathrm{~min}$ and filtered through a $0.45 \mu \mathrm{m}$ filter (Millipore, Rockland, MA). T25 flasks (Corning, Corning, NY) of HUVECs (25-30\% confluent) were transduced with $2 \mathrm{ml}$ viral supernatant, $3 \mathrm{ml}$ endothelial growth medium and polybrene $(12 \mu \mathrm{g} / \mathrm{ml}$; Sigma) for $5 \mathrm{~h}$. HUVECs were cultured for 4 days prior to use in experiments.

In overexpression studies, lentiviruses were made for Hic-5 full-length, Hic-5 $\Delta$ LIM2-3, and GFP control using $2.5 \mu \mathrm{g}$ each of backbone and $7.5 \mu \mathrm{g}$ of VIRAPOWER packaging mix as described above. After $16 \mathrm{~h}$, cells were fed with $6 \mathrm{ml}$ of growth medium, and lentiviruses were collected at 56-72 h post-transfection. Supernatants were spun at $1000 \mathrm{~g}$ for $10 \mathrm{~min}$ at room temperature and concentrated with Lenti-X-concentrator (Clontech) as per the manufacturer's instructions. HUVECs were treated with lentiviral particles for $72-96 \mathrm{~h}$ with $12 \mu \mathrm{g} / \mathrm{ml}$ polybrene. At 5 days following transduction, HUVECs were used in invasion assays as described above. Constructs expressing EGFP controls were generated as previously described (Lee et al., 2009), and protein extracts were collected to verify protein expression by performing western blotting.

\section{Generation of stable cell lines using rescue constructs}

Lenitviruses for shHic-5-1 and rescue constructs for FL-Hic-5, $\Delta$ LIM2-3Hic-5 and GFP were generated as described above. HUVECs were transduced with shHic-5-1 for $5 \mathrm{~h}$. After $30 \mathrm{~h}$, lentiviruses delivering rescue constructs to FL-Hic-5, $\Delta$ LIM2-3 Hic-5 or GFP control were added. HUVECs were treated with lentiviral particles for $72-96 \mathrm{~h}$ with $12 \mu \mathrm{g} / \mathrm{ml}$ polybrene. Cells were used in invasion assays 5 days later.

\section{Immunoblotting}

Protein samples were resolved using 8.5-14\% SDS-PAGE gels, transferred onto Immobilon PVDF membranes (Millipore), blocked with 5\% non-fat dried milk or 5\% BSA (Sigma), washed, and probed with primary antibodies overnight at $4^{\circ} \mathrm{C}$. Membranes were incubated with HRP-conjugated secondary antibodies (Dako, Carpinteria, CA), washed, and developed with Immobilon Western Chemiluminescent HRP Substrate (Millipore) and HyBlot CL autoradiography film (Denville Scientific, South Plainfield, NJ). The antibodies used were directed against Hic-5 (611164, BD Transduction Laboratories, San Jose, CA; 1:1000 dilution), MT1-MMP (MAB3328, Millipore; 1:2000 dilution), MT1-MMP phospho-tyrosine 573 (custom antisera, 21st Century Biologicals; 1:500 dilution) (Nyalendo et al., 2007), pFAK (ab4803, Abcam, Cambridge, MA; 1:1000 dilution), FAK (05-537, Millipore; 1:1000 dilution), $\beta 2 \mathrm{M}$ (M8523, Sigma; 1:500 dilution), actin (CP01, Millipore; 1:5000 dilution), $\alpha$-tubulin (T6199, Sigma; 1:10,000 dilution), GAPDH (ab8245, Abcam; 1:10,000 dilution), Flag (ab18230, Abcam or F7425, Sigma; 1:1000 dilution), integrin $\beta 1$ (610467, BD Transduction Laboratories; 1:1000 dilution), vinculin (V9131, Sigma; 1:1000 dilution), PECAM1 (sc1505, Santa Cruz Biotechnology, Temecula, CA; 1:5000 dilution), VE-cadherin (sc52751, Santa Cruz Biotechnology; 1:2000 dilution), RACK1 (sc17754, Santa Cruz Biotechnology; 1:1000 dilution), vimentin (sc5565, Santa Cruz Biotechnology; 1:1000 dilution), paxillin (sc365059, Santa Cruz Biotechnology; 1:1000 dilution), zyxin (Cell Signaling, 3553; 1:1000 dilution), and filaminA (Bethyl, A301-134A; 1:1000 dilution).

\section{Immunoprecipitation}

Endothelial cells $\left(3 \times 10^{6}\right.$ cells) cultured in $10-\mathrm{cm}$ dishes were serum starved for $4 \mathrm{~h}$ and treated with $1 \mu \mathrm{M} \mathrm{S1P}$ and $40 \mathrm{ng} / \mathrm{ml}$ VEGF and bFGF for
$30 \mathrm{~min}$. Cells were placed on ice, washed twice with $10-\mathrm{ml}$ cold PBS with cations $\left(1.5 \mathrm{mM} \mathrm{Mg}{ }^{2+}\right.$ and $\left.\mathrm{Ca}^{2+}\right)$, lysed in $700 \mu \mathrm{l}$ cold lysis buffer containing $0.5 \%$ NP-40 in PBS with cations, $1 \times$ protease inhibitor cocktail (Roche, Mannheim, Baden-Württemberg, Germany) and 100× HALT phosphatase inhibitor (Thermo Scientific, Ashville, NC), and incubated for $10 \mathrm{~min}$ on ice with occasional mixing. Lysates were centrifuged at $16,000 \mathrm{~g}$ for $15 \mathrm{~min}$ at $4^{\circ} \mathrm{C}$. Supernatants were collected and precleared with proteinG-conjugated magnetic beads (Invitrogen) $(5 \mu \mathrm{l})$ for $1 \mathrm{~h}$ at $4^{\circ} \mathrm{C}$ with agitation. Supernatants were incubated with $2 \mu \mathrm{g}$ antisera directed against MT1-MMP (ab38971, Abcam) or FAK (ab40794, Abcam) or speciesspecific IgG control for $18 \mathrm{~h}$ at $4^{\circ} \mathrm{C}$ with agitation. Protein-G-conjugated magnetic beads $(10 \mu \mathrm{l})$ were added for $2 \mathrm{~h}$ at $4^{\circ} \mathrm{C}$. Beads were washed five times with $1 \mathrm{ml}$ lysis buffer without protease inhibitors, eluted in $1 \%$ SDS and analyzed by performing western blotting.

\section{Immunofluorescence}

Endothelial cells were seeded on collagen-coated coverslips $(50 \mu \mathrm{g} / \mathrm{ml})$ overnight. Cells were washed twice with M199 and serum starved for $2 \mathrm{~h}$. Cells were treated with $1 \mu \mathrm{M} \mathrm{S1P}$ or $40 \mathrm{ng} / \mathrm{ml}$ growth factors (or both), fixed in 4\% paraformaldehyde (Electron Microscopy Sciences, Hatfield, PA), rinsed three times in Tris-glycine buffer $(0.3 \%$ Tris and $1.5 \%$ glycine $)$, and permeabilized with $0.5 \%$ Triton X-100 (Sigma) for 20 min with gentle agitation before adding blocking buffer $(0.5 \%$ Triton X-100, $1 \%$ BSA and $1 \%$ serum) overnight at $4{ }^{\circ} \mathrm{C}$. Primary antibodies were added in blocking buffer (1:100) for $3 \mathrm{~h}$ at room temperature, washed, and incubated with Alexa-Fluor488- or 594-conjugated secondary antibodies (Molecular Probes, Grand Island, NY, USA) (1:300) in blocking buffer for $1 \mathrm{~h}$. After washing, coverslips were mounted and imaged using a Nikon TI A1R inverted confocal microscope (Yurakucho, Tokyo, Japan). Primary antibodies used for staining were directed against Hic-5 (611164, BD Transduction Laboratories), pFAK (Tyr397, ab4803, Abcam) and VE-cadherin (sc9989, Santa Cruz Biotechnology). Antibodies recognizing phosphorylated MT1-MMP (Tyr573) and pre-immune serum were obtained from 21st Century Biochemicals (Marlborough, MA), as previously described (Langlois et al., 2004). Cross sections of invading cultures were generated by allowing endothelial cells to invade collagen matrices for $72 \mathrm{~h}$ prior to fixing in $4 \%$ paraformaldehyde. Invasion cultures were embedded in Tissue-Tek OCT compound (Sakura Finetek, Torrance, CA) frozen in liquid nitrogen, and stored at $-80^{\circ} \mathrm{C}$. Frozen sections $(20 \mu \mathrm{m})$ were fixed with methanol at $-20^{\circ} \mathrm{C}$ for $15 \mathrm{~min}$, and then washed three times for $5 \mathrm{~min}$ in PBS at room temperature. These sections were blocked in $10 \%$ goat serum (Invitrogen) for $2 \mathrm{~h}$ at room temperature. For double-staining, primary antibodies $(4 \mu \mathrm{g} / \mathrm{ml})$ were added and incubated overnight at $4^{\circ} \mathrm{C}$ in a humidified chamber. The primary antibodies used for this study were: Hic-5 (611164, BD Transduction Laboratories), MT1-MMP (MAB3328, Millipore), pMT1-MMP (21st Century Biochemicals), FAK (ab40794, Abcam), pFAK (Tyr397, ab4803, Abcam), and VE-cadherin (ALX-210-232-C100, Enzo Life Sciences, Farmingdale, NY). Tissue sections were washed five times for $20 \mathrm{~min}$ in PBS. The goat anti-rabbit-IgG conjugated to Alexa Fluor 488 and goat antimouse-IgG conjugated to Alexa Fluor 594 (Molecular Probes) were added and incubated for $1 \mathrm{~h}$ at room temperature and washed six times for $20 \mathrm{~min}$ in PBS. Slides were counterstained with Prolong Gold Antifade reagent with DAPI (Life Technologies, Grand Island, NY; 1:500) and coverslips were added. For in vivo studies, samples were used from a previously conducted study (Song et al., 2010). Sexually mature gilts of similar age, weight and genetic background (F1 crosses of Yorkshire X Landrace sows and Duroc X Hampshire boars) were observed daily for estrus (day 0 ) and exhibited at least two estrus cycles of normal duration (18 to 21 days) before being used in these studies. All experimental and surgical procedures were in compliance with the Guide for Care and Use of Agricultural Animals in Teaching and Research and approved by the Institutional Animal Care and Use Committee of Texas A\&M University (AUP 2006-114). Gilts were ovariohysterectomized on day 24 of pregnancy, and several sections $(\sim 0.5 \mathrm{~cm})$ from the middle of each uterine horn were embedded in Paraplast-Plus (Oxford Laboratory, St Louis, MO). Frozen sections $(10 \mu \mathrm{m})$ were prepared from three randomly selected animals and stained as described above. All image stacks were collected using Nikon A1 confocal laser microscopy. 


\section{Isolation of DRM fractions from endothelial cells}

Endothelial cells (30,000 cells/well) were allowed to invade for $6 \mathrm{~h}$ before collagen matrices were washed three times with $200 \mu$ cold PBS with cations. Starting material was collected by solubilizing collagen matrices from each treatment group into boiling $1.5 \times$ sample buffer $(50 \mu \mathrm{l} / \mathrm{gel}) .100 \mu \mathrm{l}$ of CSK buffer [10 mM HEPES, $250 \mathrm{mM}$ sucrose, $150 \mathrm{mM} \mathrm{KCl,} 3 \mathrm{mM}$ $\mathrm{MgCl}_{2}, 1 \mathrm{mM}$ EGTA, $1 \times$ protease inhibitor cocktail (Roche), 100× HALT phosphatase inhibitor] containing $0.05 \%, 0.1 \%$ or $0.5 \%$ Triton $\mathrm{X}-100$ was added consecutively to each collagen matrix and allowed to incubate on ice for $10 \mathrm{~min}$ with continuous, gentle agitation. Soluble fractions were collected after each incubation. After the final wash, DRM fractions were collected by solubilizing the remaining material in $1.5 \times$ boiling sample buffer $(30 \mu \mathrm{l} / \mathrm{gel})$. Starting material, detergent soluble fractions, and DRM fractions were analyzed by performing western blotting.

\section{Surface biotinylation assays}

Three days after lentiviral transduction with shRNA directed against $\beta 2 \mathrm{M}$ or Hic-5, cells were seeded into six-well plates coated with $50 \mu \mathrm{g} / \mathrm{ml}$ collagen. The next day, cells were serum starved for $30 \mathrm{~min}$ and treated with $1 \mu \mathrm{M} \mathrm{S} 1 \mathrm{P}$ for 0,15 or $60 \mathrm{~min}$. The plate was placed on ice and each well washed two times with ice-cold PBS with cations. $1 \mathrm{ml}$ cell-impermeable biotin (EZ-Link sulfo-NHS-LC-LC-biotin, Sigma) at $0.5 \mathrm{mg} / \mathrm{ml}$ in PBS with cations was added to each well and the plate was rotated on ice for $30 \mathrm{~min}$. After rinsing wells two times with ice-cold PBS with cations, wells were rinsed with $100 \mathrm{mM}$ glycine (Sigma) in PBS with cations. Cells were lysed in $450 \mu \mathrm{l}$ buffer containing 1\% NP-40, 0.5\% sodium deoxycholate, $1 \times$ protease inhibitors (Roche) and $1 \times$ Halt phosphatase inhibitor in TBS. Lysates were collected and centrifuged at $10,000 \mathrm{~g}$ for $10 \mathrm{~min}$, at $4^{\circ} \mathrm{C}$. Equal amounts of lysate from each treatment group were added to $30 \mu \mathrm{lhigh}-$ capacity Neutravidin resin beads (Thermo Scientific, Waltham, MA) and incubated, rotating at $4^{\circ} \mathrm{C}$ for $16 \mathrm{~h}$. Beads were washed four times with PBS, and bound proteins were eluted in boiling sample buffer. After western blotting, the MT1-MMP band intensity values were determined using ImageJ software and normalized to the PECAM1 band intensity for each respective sample. MT1-MMP fold change expression at cell surface relative to no S1P treatment was calculated from normalized MT1-MMP band intensities. Three independent experiments were combined and quantified.

\section{MT1-MMP activity assays}

MT1-MMP activity assays (AS-72025, Sensolyte) were performed by using invading endothelial cells that had been stimulated for $6 \mathrm{~h}$ with S1P and growth factors. Wells were washed with $100 \mu \mathrm{PBS}$ with cations. Collagen matrices were added to $50 \mu \mathrm{l}$ Component D with $0.1 \%$ Triton X-100 for $10 \mathrm{~min}$, vortexing every $3 \mathrm{~min}$. Cleared supernatants were added to black 96-well plates and incubated with conditioned medium collected from TIMP-1-transfected HEK-293 cells to inhibit soluble MMPs, as previously described (Kwak et al., 2012). The MT1-MMP-sensitive substrate was added to the wells, mixed briefly and incubated at $37^{\circ} \mathrm{C}$ for $30 \mathrm{~min}$. Fluorescence intensity was measured at an excitation of $490 \mathrm{~nm}$ and emission of $520 \mathrm{~nm}$ using a Victor X3 Multilabel Reader 2030. Relative fluorescence units (RFUs) were calculated by subtracting the control reading (no cell lysate) from each sample, and normalizing the total protein content between samples. Values were averaged to generate the RFU for each treatment group.

\section{Competing interests}

The authors declare no competing or financial interests.

\section{Author contributions}

Endothelial experiments were performed by J.M.D., C.A.A., C.L.D. and K.J.B. Porcine tissue staining was conducted by H.S. Data analysis was carried out by J.M. D., C.A.A., C.L.D., H.S., G.A.J. and K.J.B. J.M.D., C.A.A., C.L.D. and K.J.B. contributed to the preparation of the manuscript.

\section{Funding}

This work was supported by the Public Health Service [grant number HL-095786 to K.J.B.]; and the National Institutes of Health [grant number HD-071468 to G.A.J. and K.J.B.]. Deposited in PMC for release after 12 months.
Supplementary information

Supplementary information available online at

http://jcs.biologists.org/lookup/suppl/doi:10.1242/jcs.170571/-/DC1

\section{References}

Adams, R. H. and Alitalo, K. (2007). Molecular regulation of angiogenesis and lymphangiogenesis. Nat. Rev. Mol. Cell. Biol. 8, 464-478.

Ambati, J. and Fowler, B. J. (2012). Mechanisms of age-related macular degeneration. Neuron 75, 26-39.

Artym, V. V., Zhang, Y., Seillier-Moiseiwitsch, F., Yamada, K. M. and Mueller, S. C. (2006). Dynamic interactions of cortactin and membrane type 1 matrix metalloproteinase at invadopodia: defining the stages of invadopodia formation and function. Cancer Res. 66, 3034-3043.

Avraamides, C., Bromberg, M. E., Gaughan, J. P., Thomas, S. M., Tsygankov, A. Y. and Panetti, T. S. (2007). Hic-5 promotes endothelial cell migration to lysophosphatidic acid. Am. J. Physiol. Heart Circ. Physiol. 293, H193-H203.

Azizi, G., Boghozian, R. and Mirshafiey, A. (2014). The potential role of angiogenic factors in rheumatoid arthritis. Int. J. Rheum. Dis. 17, 369-383.

Bayless, K. J., Kwak, H.-I. and Su, S.-C. (2009). Investigating endothelial invasion and sprouting behavior in three-dimensional collagen matrices. Nat. Protoc. 4 1888-1898.

Brown, M. C. and Turner, C. E. (2004). Paxillin: adapting to change. Physiol. Rev. 84, 1315-1339.

Brown, M. C., Perrotta, J. A. and Turner, C. E. (1996). Identification of LIM3 as the principal determinant of paxillin focal adhesion localization and characterization of a novel motif on paxillin directing vinculin and focal adhesion kinase binding J. Cell Biol 135, 1109-1123.

Carmeliet, P. (2003). Angiogenesis in health and disease. Nat. Med. 9, 653-660.

Chen, L. M., Bailey, D. and Fernandez-Valle, C. (2000). Association of beta 1 integrin with focal adhesion kinase and paxillin in differentiating Schwann cells. J. Neurosci. 20, 3776-3784.

Chun, T.-H., Sabeh, F., Ota, l., Murphy, H., McDonagh, K. T., Holmbeck, K., Birkedal-Hansen, H., Allen, E. D. and Weiss, S. J. (2004). MT1-MMPdependent neovessel formation within the confines of the three-dimensional extracellular matrix. J. Cell Biol. 167, 757-767.

Cukierman, E., Pankov, R., Stevens, D. R. and Yamada, K. M. (2001). Taking cellmatrix adhesions to the third dimension. Science 294, 1708-1712.

Dave, J. M., Kang, H., Abbey, C. A., Maxwell, S. A. and Bayless, K. J. (2013). Proteomic profiling of endothelial invasion revealed receptor for activated $\mathrm{C}$ kinase 1 (RACK1) complexed with vimentin to regulate focal adhesion kinase (FAK). J. Biol. Chem. 288, 30720-30733

Davis, G. E., Stratman, A. N., Sacharidou, A. and Koh, W. (2011). Molecular basis for endothelial lumen formation and tubulogenesis during vasculogenesis and angiogenic sprouting. Int. Rev. Cell Mol. Biol. 288, 101-165.

Dawid, I. B., Toyama, R. and Taira, M. (1995). LIM domain proteins. C. R. Acad. Sci. III 318, 295-306

Deakin, N. O. and Turner, C. E. (2008). Paxillin comes of age. J. Cell Sci. 121 2435-2444.

Deakin, N. O. and Turner, C. E. (2011). Distinct roles for paxillin and Hic-5 in regulating breast cancer cell morphology, invasion, and metastasis. Mol. Biol. Cell 22, 327-341.

Deakin, N. O., Ballestrem, C. and Turner, C. E. (2012). Paxillin and Hic-5 interaction with vinculin is differentially regulated by Rac1 and RhoA. PLOS ONE 7, e37990

Egeblad, M. and Werb, Z. (2002). New functions for the matrix metalloproteinases in cancer progression. Nat. Rev. Cancer 2, 161-174.

Folkman, J. (1995). Angiogenesis in cancer, vascular, rheumatoid and other disease. Nat. Med. 1, 27-30.

Folkman, J. and D'Amore, P. A. (1996). Blood vessel formation: what is its molecular basis? Cell 87, 1153-1155

Fujita, H., Kamiguchi, K., Cho, D., Shibanuma, M., Morimoto, C. and Tachibana, K. (1998). Interaction of Hic-5, A senescence-related protein, with focal adhesion kinase. J. Biol. Chem. 273, 26516-26521.

Gingras, D., Michaud, M., Di Tomasso, G., Béliveau, E., Nyalendo, C. and Béliveau, R. (2008). Sphingosine-1-phosphate induces the association of membrane-type 1 matrix metalloproteinase with p130Cas in endothelial cells FEBS Lett. 582, 399-404.

Guan, J.-L. (1997). Focal adhesion kinase in integrin signaling. Matrix Biol. 16 195-200

Hiraoka, N., Allen, E., Apel, I. J., Gyetko, M. R. and Weiss, S. J. (1998). Matrix metalloproteinases regulate neovascularization by acting as pericellula fibrinolysins. Cell 95, 365-377.

Holmbeck, K., Bianco, P., Caterina, J., Yamada, S., Kromer, M., Kuznetsov, S. A., Mankani, M., Gehron Robey, P., Poole, A. R., Pidoux, I. et al. (1999). MT1-MMP-deficient mice develop dwarfism, osteopenia, arthritis, and connective tissue disease due to inadequate collagen turnover. Cell 99, 81-92.

Hotary, K., Allen, E., Punturieri, A., Yana, I. and Weiss, S. J. (2000). Regulation of cell invasion and morphogenesis in a three-dimensional type I collagen matrix 
by membrane-type matrix metalloproteinases 1, 2, and 3. J. Cell Biol. 149, 1309-1323.

Iruela-Arispe, M. L. and Davis, G. E. (2009). Cellular and molecular mechanisms of vascular lumen formation. Dev. Cell 16, 222-231.

Jia, Y., Ransom, R. F., Shibanuma, M., Liu, C., Welsh, M. J. and Smoyer, W. E. (2001). Identification and characterization of hic-5/ARA55 as an hsp27 binding protein. J. Biol. Chem. 276, 39911-39918.

Kim-Kaneyama, J. R. (2012). [Hydrogen peroxide-inducible clone 5 (Hic-5) as a potential therapeutic target]. Seikagaku 84, 261-265.

Kim-Kaneyama, J.-r., Suzuki, W., Ichikawa, K., Ohki, T., Kohno, Y., Sata, M., Nose, K. and Shibanuma, M. (2005). Uni-axial stretching regulates intracellular localization of Hic-5 expressed in smooth-muscle cells in vivo. J. Cell Sci.118, 937-949

Kim-Kaneyama, J.-r., Takeda, N., Sasai, A., Miyazaki, A., Sata, M., Hirabayashi, T., Shibanuma, M., Yamada, G. and Nose, K. (2011). Hic-5 deficiency enhances mechanosensitive apoptosis and modulates vascular remodeling. J. Mol. Cell. Cardiol. 50, 77-86.

Kwak, H.-I., Kang, H., Dave, J. M., Mendoza, E. A., Su, S.-C., Maxwell, S. A. and Bayless, K. J. (2012). Calpain-mediated vimentin cleavage occurs upstream of MT1-MMP membrane translocation to facilitate endothelial sprout initiation. Angiogenesis 15, 287-303

Labrecque, L., Nyalendo, C., Langlois, S., Durocher, Y., Roghi, C., Murphy, G., Gingras, D. and Béliveau, R. (2004). Src-mediated tyrosine phosphorylation of caveolin-1 induces its association with membrane type 1 matrix metalloproteinase. J. Biol. Chem. 279, 52132-52140.

Langlois, S., Gingras, D. and Béliveau, R. (2004). Membrane type 1-matrix metalloproteinase (MT1-MMP) cooperates with sphingosine 1-phosphate to induce endothelial cell migration and morphogenic differentiation. Blood 103 , 3020-3028

Lee, P.-F., Yeh, A. T. and Bayless, K. J. (2009). Nonlinear optical microscopy reveals invading endothelial cells anisotropically alter three-dimensional collagen matrices. Exp. Cell Res. 315, 396-410.

Li, X.-Y., Ota, I., Yana, I., Sabeh, F. and Weiss, S. J. (2008). Molecular dissection of the structural machinery underlying the tissue-invasive activity of membrane type1 matrix metalloproteinase. Mol. Biol. Cell 19, 3221-3233.

Matsuya, M., Sasaki, H., Aoto, H., Mitaka, T., Nagura, K., Ohba, T., Ishino, M., Takahashi, S., Suzuki, R. and Sasaki, T. (1998). Cell adhesion kinase beta forms a complex with a new member, Hic-5, of proteins localized at focal adhesions. J. Biol. Chem. 273, 1003-1014.

Murphy, G. and Gavrilovic, J. (1999). Proteolysis and cell migration: creating a path? Curr. Opin. Cell Biol. 11, 614-621.

Nishiya, N., Iwabuchi, Y., Shibanuma, M., Cote, J.-F., Tremblay, M. L. and Nose, K. (1999). Hic-5, a Paxillin homologue, binds to the protein-tyrosine phosphatase PEST (PTP-PEST) through its LIM 3 domain. J. Biol. Chem. 274, 9847-9853.

Nishiya, N., Shirai, T., Suzuki, W. and Nose, K. (2002). Hic-5 interacts with GIT1 with a different binding mode from paxillin. J. Biochem. 132, 279-289.

Nyalendo, C., Michaud, M., Beaulieu, E., Roghi, C., Murphy, G., Gingras, D. and Beliveau, R. (2007). Src-dependent phosphorylation of membrane type I matrix metalloproteinase on cytoplasmic tyrosine 573: role in endothelial and tumor cell migration. J. Biol. Chem. 282, 15690-15699.

Osenkowski, P., Toth, M. and Fridman, R. (2004). Processing, shedding, and endocytosis of membrane type 1-matrix metalloproteinase (MT1-MMP). J. Cell Physiol. 200, 2-10.

Peng, X., Ueda, H., Zhou, H., Stokol, T., Shen, T. L., Alcaraz, A., Nagy, T., Vassalli, J. D. and Guan, J. L. (2004). Overexpression of focal adhesion kinase in vascular endothelial cells promotes angiogenesis in transgenic mice. Cardiovasc. Res. 64, 421-430.

Petroll, W. M. and Ma, L. (2003). Direct, dynamic assessment of cell-matrix interactions inside fibrillar collagen lattices. Cell Motil. Cytoskeleton 55, 254-264

Pignatelli, J., Tumbarello, D. A., Schmidt, R. P. and Turner, C. E. (2012). Hic-5 promotes invadopodia formation and invasion during TGF-beta-induced epithelial-mesenchymal transition. J. Cell Biol. 197, 421-437.

Remacle, A., Murphy, G. and Roghi, C. (2003). Membrane type I-matrix metalloproteinase (MT1-MMP) is internalised by two different pathways and is recycled to the cell surface. J. Cell Sci. 116, 3905-3916.

Remacle, A. G., Rozanov, D. V., Fugere, M., Day, R. and Strongin, A. Y. (2006) Furin regulates the intracellular activation and the uptake rate of cell surfaceassociated MT1-MMP. Oncogene 25, 5648-5655.

Sacharidou, A., Koh, W., Stratman, A. N., Mayo, A. M., Fisher, K. E. and Davis, G. E. (2010). Endothelial lumen signaling complexes control 3D matrix-specific tubulogenesis through interdependent Cdc42- and MT1-MMP-mediated events. Blood 115, 5259-5269.

Senger, P. L. (2011). In Pathways to Pregnancy and Parturition (ed. P. L. Senger) Current Conceptions Inc.
Shen, T.-L., Park, A. Y.-J., Alcaraz, A., Peng, X., Jang, I., Koni, P., Flavell, R. A. Gu, H. and Guan, J.-L. (2005). Conditional knockout of focal adhesion kinase in endothelial cells reveals its role in angiogenesis and vascular development in late embryogenesis. J. Cell Biol. 169, 941-952.

Shibanuma, M., Mashimo, J., Kuroki, T. and Nose, K. (1994). Characterization of the TGF beta 1-inducible hic-5 gene that encodes a putative novel zinc finge protein and its possible involvement in cellular senescence. J. Biol. Chem. 269 26767-26774.

Shibanuma, M., Iwabuchi, Y. and Nose, K. (2002). Possible involvement of hic-5, a focal adhesion protein, in the differentiation of $\mathrm{C} 2 \mathrm{C} 12$ myoblasts. Cell Struct. Funct. 27, 21-27.

Shibanuma, M., Kim-Kaneyama, J. R., Ishino, K., Sakamoto, N., Hishiki, T., Yamaguchi, K., Mori, K., Mashimo, J.-i. and Nose, K. (2003). Hic-5 communicates between focal adhesions and the nucleus through oxidantsensitive nuclear export signal. Mol. Biol. Cell 14, 1158-1171.

Shibanuma, M., Kim-Kaneyama, J.-r., Sato, S. and Nose, K. (2004). A LIM protein, Hic-5, functions as a potential coactivator for Sp1. J. Cell. Biochem. 91 633-645.

Shibanuma, M., Mori, K. and Nose, K. (2012). HIC-5: a mobile molecular scaffold regulating the anchorage dependence of cell growth. Int. J. Cell Biol. 2012 426138.

Song, G., Bailey, D. W., Dunlap, K. A., Burghardt, R. C., Spencer, T. E., Bazer, F. W. and Johnson, G. A. (2010). Cathepsin B, cathepsin L, and cystatin C in the porcine uterus and placenta: potential roles in endometrial/placental remodeling and in fluid-phase transport of proteins secreted by uterine epithelia across placental areolae. Biol. Reprod. 82, 854-864

Stratman, A. N., Saunders, W. B., Sacharidou, A., Koh, W., Fisher, K. E. Zawieja, D. C., Davis, M. J. and Davis, G. E. (2009). Endothelial cell lumen and vascular guidance tunnel formation requires MT1-MMP-dependent proteolysis in 3-dimensional collagen matrices. Blood 114, 237-247.

Takino, T., Watanabe, Y., Matsui, M., Miyamori, H., Kudo, T., Seiki, M. and Sato, H. (2006). Membrane-type 1 matrix metalloproteinase modulates focal adhesion stability and cell migration. Exp. Cell Res. 312, 1381-1389.

Takino, T., Saeki, H., Miyamori, H., Kudo, T. and Sato, H. (2007). Inhibition of membrane-type 1 matrix metalloproteinase at cell-matrix adhesions. Cancer Res. 67, 11621-11629.

Thomas, S. M., Hagel, M. and Turner, C. E. (1999). Characterization of a focal adhesion protein, Hic-5, that shares extensive homology with paxillin. J. Cell Sci. 112, 181-190.

Tumbarello, D. A. and Turner, C. E. (2007). Hic-5 contributes to epithelialmesenchymal transformation through a RhoA/ROCK-dependent pathway. J. Cell Physiol. 211, 736-747.

Uekita, T., Itoh, Y., Yana, I., Ohno, H. and Seiki, M. (2001). Cytoplasmic taildependent internalization of membrane-type 1 matrix metalloproteinase is important for its invasion-promoting activity. J. Cell Biol. 155, 1345-1356.

Wang, Y. and McNiven, M. A. (2012). Invasive matrix degradation at focal adhesions occurs via protease recruitment by a FAK-p130Cas complex. J. Cell Biol. 196, 375-385.

Wang, R., Li, Q.-F., Anfinogenova, Y. and Tang, D. D. (2007). Dissociation of Crkassociated substrate from the vimentin network is regulated by p21-activated kinase on ACh activation of airway smooth muscle. Am. J. Physiol. Lung Cell. Mol. Physiol. 292, L240-L248.

Werb, Z. (1997). ECM and cell surface proteolysis: regulating cellular ecology. Cell 91, 439-442.

Wu, R. F., Xu, Y. C., Ma, Z., Nwariaku, F. E., Sarosi, G. A., Jr. and Terada, L. S. (2005a). Subcellular targeting of oxidants during endothelial cell migration. J. Cell Biol. 171, 893-904.

Wu, X., Gan, B., Yoo, Y. and Guan, J.-L. (2005b). FAK-mediated src phosphorylation of endophilin A2 inhibits endocytosis of MT1-MMP and promotes ECM degradation. Dev. Cell 9, 185-196.

Yana, I. and Weiss, S. J. (2000). Regulation of membrane type-1 matrix metalloproteinase activation by proprotein convertases. Mol. Biol. Cell 11 2387-2401.

Yana, I. and Seiki, M. (2003). [Matrix metalloproteinases; the past, present, and future]. Nihon Shokakibyo Gakkai Zasshi 100, 144-151.

Yana, I., Sagara, H., Takaki, S., Takatsu, K., Nakamura, K., Nakao, K., Katsuki, M., Taniguchi, S.-i., Aoki, T., Sato, H. et al. (2007). Crosstalk between neovessels and mural cells directs the site-specific expression of MT1-MMP to endothelial tip cells. J. Cell Sci. 120, 1607-1614.

Zhou, Z., Apte, S. S., Soininen, R., Cao, R., Baaklini, G. Y., Rauser, R. W., Wang J., Cao, Y. and Tryggvason, K. (2000). Impaired endochondral ossification and angiogenesis in mice deficient in membrane-type matrix metalloproteinase I. Proc. Natl. Acad. Sci. USA 97, 4052-4057. 